\title{
Volume of a vortex and the Bradlow bound
}

\author{
C. Adam, ${ }^{1}$ J. M. Speight, ${ }^{2}$ and A. Wereszczynski ${ }^{3}$ \\ ${ }^{1}$ Departamento de Física de Partículas, Universidad de Santiago de Compostela and Instituto Galego de \\ Física de Altas Enerxias (IGFAE), E-15782 Santiago de Compostela, Spain \\ ${ }^{2}$ School of Mathematics, University of Leeds, Leeds LS2 9JT, United Kingdom \\ ${ }^{3}$ Institute of Physics, Jagiellonian University, Lojasiewicza 11, Kraków 30-348, Poland
}

(Received 30 March 2017; published 16 June 2017)

\begin{abstract}
We demonstrate that the geometric volume of a soliton coincides with the thermodynamical volume also for field theories with higher-dimensional vacuum manifolds (e.g., for gauged scalar field theories supporting vortices or monopoles), generalizing the recent results of Ref. [C. Adam, M. Haberichter, and A. Wereszczynski, Phys. Lett. B 754, 18 (2016).]. We apply this observation to understand Bradlowtype bounds for general Abelian gauge theories supporting vortices, as well as some thermodynamical aspects of said theories. In the case of SDiff Bogomolny-Prasad-Sommerfield (BPS) models (being examples of perfect fluid models) we show that the base-space independent geometric volume (area) of the vortex is exactly equal to the Bradlow volume (a minimal volume for which BPS soliton solutions exist). This volume can be finite for compactons or infinite for infinitely extended solitons (in flat Minkowski space-time).
\end{abstract}

DOI: $10.1103 /$ PhysRevD.95.116007

\section{INTRODUCTION}

Solitons are nonperturbative solutions of nonlinear field theories that behave like particles in many respects. They may be viewed as collective excitations of the underlying basic fields and are, therefore, particularly suitable for the description of collective phenomena. Topological solitons [1], additionally, allow us to define a (typically integervalued) topological index, resulting either from the topology of the base space or, for Euclidean base space $\mathbb{R}^{d}$, from the condition of finite energy. This topological index provides a natural definition for a conserved particle number (soliton number). Apart from their independent relevance as interesting mathematical structures, field theories supporting topological solitons are frequently employed as low-energy effective field theories (LE-EFTs) of some underlying fundamental systems (e.g., UV field theories, or manyparticle systems). Here, the basic fields of the solitonic LE-EFT describe the most important degrees of freedom (DoF) at low energies, and the solitons correspond to certain collective excitations of said basic DoF. Examples thereof are, e.g., the Skyrme model [2] as a LE-EFT for the strong interactions (with mesons as basic fields and baryons as solitonic excitations), or the Ginzburg-Landau theory of superconductivity [3] (with a complex scalar condensate related to Cooper pairs as the basic field and vortices as solitonic excitations). In line with their role as LE-EFT, these solitonic models should be capable of describing the macroscopic (fluid dynamical or thermodynamcial) behavior of the underlying fundamental system, at least at sufficiently low energies (particularly at zero temperature). In particular, the solitonic EFT should provide natural definitions for certain thermodynamical variables like the free energy $F$ (which coincides with the static energy $E$ at zero temperature), the pressure $P$, the volume $V$, the particle number $N$, and the chemical potential $\mu$. Like all field theories, a solitonic EFT is equipped with a natural definition of pressure (the fieldtheoretic pressure; see below). As demonstrated recently in [4], this directly implies a unique definition of the thermodynamical volume (for the case of Skyrmions, see also [5,6]). It turns out that the thermodynamical volume is equal to the geometric volume, i.e., the volume of the region in space where the static energy density is different from 0 (see also $\mathrm{Sec}$. II). Further, as mentioned already, the topological index of topological solitons implies a natural definition of particle number. The corresponding chemical potential may then be found rather straightforwardly (see [7] for the case of skyrmions).

The fact that the thermodynamical volume $V_{\mathrm{t}}$ equals the geometric volume $V_{\mathrm{g}}$ implies some consequences that might seem surprising at first sight. While solitons at finite pressure always occupy a finite volume, the volume of a soliton at zero pressure may be finite (a compacton) or infinite. In particular, the well-known examples of solitons with exponential tails lead to infinite thermodynamical volume. This finding may be less surprising once one interprets a solitonic model as a LE-EFT. If a solitonic EFT is, e.g., supposed to describe a many-particle system of identical fermions, then a nonzero pressure (Fermi pressure) remains at zero temperature, which must be balanced (by an external pressure) to maintain the system in a finite volume. In the zero-pressure limit, the system tends to infinite volume at equilibrium. There exist, of course, further definitions for the volume of a soliton (e.g., based on its mean-square radius) that typically lead to finite values for the volume of a soliton with exponential tail, and which have their own applications. 
These volume definitions, however, cannot be used as thermodynamical volumes.

Both the characteristics of solitonic solutions and their thermodynamical properties may undergo significant changes when they are considered on a base-space manifold $\mathcal{M}$ with finite volume (e.g., a compact manifold) instead of flat space $\mathbb{R}^{d}$. One might, e.g., naively suppose that an infinitely extended soliton will always lead to nonzeropressure solutions on a compact base space, but this is not necessarily true. Depending on the boundary conditions implied by the base space topology, infinitely extended solitons on $\mathbb{R}^{d}$ may, nevertheless, "fit" on a compact base space. The investigation of these issues is simplified significantly in the case of so-called Bogomolny-PrasadSommerfield (BPS) field theories, i.e., field theories that allow us to reduce the static field equations (Euler-Lagrange equations) to first-order equations (the BPS equations) $[8,9]$ such that the resulting BPS solutions saturate a global (topological) energy bound. A BPS solution has zero pressure, by definition (see Sec. II), so for BPS models the search for zero-pressure solutions on compact spaces is equivalent to the existence of BPS solutions on the same spaces. In the case of BPS vortices, their existence is related to the so-called Bradlow bound [10], i.e., a minimum volume (area) that a compact base-space manifold must have in order to support BPS vortices. It is the main purpose of the present paper to study this Bradlow bound and, generally, the effect of finite volume base spaces on BPS soliton solutions. We, therefore, restrict ourselves to the case of vortices and their Bradlow bounds in the rest of the paper, although bounds equivalent to the Bradlow bound may probably be defined also for other types of topological solitons (e.g., monopoles).

The presumably best-known field theory supporting vortices of finite energy is the Abelian-Higgs model [11], whose vortex solutions have been analyzed in [12]. The static sector coincides with the Ginzburg-Landau theory of superconductivity [3], where the corresponding vortex solutions are known as Abrikosov vortices [13]. Vortices also play a role as magnetic domains in magnetic materials and may show up in Bose condensates and superfluidity, and they make their appearance in cosmology in the form of codimension two defects (cosmic strings), demonstrating both their ubiquitous character and their relevance.

In this paper, two properties of vortices are of special importance. The first one is the BPS property already mentioned above. Physically, the BPS property means that a multivortex BPS configuration (with "topological index" equal to $N$, say) may be interpreted as a collection of $N$ basic BPS vortices such that the forces between these basic vortices are exactly 0 . The second issue is related to the macroscopic or "thermodynamical" behavior of multivortex configurations, in general (i.e., not necessarily BPS ones). Indeed, a multivortex configuration may, e.g., behave like a gas of (interacting or noninteracting) vortices, or like a fluid.
To study these questions and to calculate average (thermodynamical) quantities, a well-defined notion of the thermodynamical volume (area for the particular case of vortices) of a multisoliton (multivortex) is required. It is one of the main objectives of the present paper to provide this notion, generalizing the results of [4] to the case of vortices (to solitons with a higher-dimensional vacuum manifold, in general). Further, in the case of vortices a related feature is the Bradlow bound mentioned above.

Concretely, in Sec. II, we provide the definition for the thermodynamical volume of a soliton, generalizing the results of [4]. In Sec. III, we briefly review the Bradlow bound and zero-temperature thermodynamics (fluid dynamics) of the standard Abelian-Higgs model, and then study the same issues for certain generalized Abelian-Higgs models. In Sec. IV, we repeat this analysis for field theories of the perfect fluid type and, in Sec. V, for their gauged versions (SDiff Abelian vortices). In Sec. VI, we briefly discuss the case of conformal solitons. Finally, Sec. VII contains our conclusions.

The field theories we consider are always defined on a space-time $\mathbb{R} \times \mathcal{M}$ with metric

$$
d s^{2}=d t^{2}-g_{i j} d x^{i} d x^{j}
$$

for a (positive definite) Riemannian metric $g_{i j}$. Further, the kinetic energy expressions of all our field theories are quadratic in momenta such that, in the static case (which is the case of interest here), the (non-negative) energy density is just minus the static Lagrangian density, $\mathcal{E}=-\mathcal{L}$, and the strain tensor is

$$
T^{i j}(x)=-\frac{2}{\sqrt{g}} \frac{\delta}{\delta g_{i j}(x)} E
$$

where $E=\int d^{d} x \sqrt{g} \mathcal{E}$ is the energy and $g=\operatorname{det} g_{i j}$ is the determinant of the metric. Further, we always assume that some units of length and energy have been fixed, such that all constants appearing in the field equations or in the soliton solutions are dimensionless.

In principle, we have to distinguish four different notions of volume in the considerations that follow. The first one is the volume $V_{\mathcal{M}}$ of the space manifold $\mathcal{M}$. The second is the Bradlow volume $V_{B}$ (the minimum volume that a space manifold must have such that it supports BPS solutions of a given field theory; obviously, $V_{B}$ depends on the field theory under consideration). The third volume is the geometric volume $V_{\mathrm{g}}$ of the soliton, i.e., the volume of the region where its energy density is nonzero. The fourth volume is the thermodynamic volume $V_{\mathrm{t}}$, defined by the (zero-temperature) thermodynamic relation

$$
P_{\mathrm{t}}=-\frac{d E}{d V_{\mathrm{t}}},
$$


where $E$ and $P_{\mathrm{t}}$ are the energy and the (thermodynamical) pressure. It turns out, however, that $V_{\mathrm{g}}$ and $V_{\mathrm{t}}$ may always naturally be assumed to be equal; therefore, we use the common symbol $V=V_{\mathrm{g}}=V_{\mathrm{t}}$. In principle, we also have to distinguish two notions of pressure, namely, the thermodynamical pressure $P_{\mathrm{t}}$ defined by the relation (1.3) and the field-theoretic pressure

$$
P_{\mathrm{f}}=\frac{1}{V_{\mathrm{g}}} \int d^{d} x \sqrt{g} \mathcal{P}, \quad \mathcal{P}=\frac{1}{d} g_{i j} T^{i j},
$$

where $\mathcal{P}$ is the pressure density. We find, however, that precisely when $V=V_{\mathrm{g}}=V_{\mathrm{t}}$ is assumed, we find as a result that the two pressures are equal and we may use the common symbol $P=P_{\mathrm{t}}=P_{\mathrm{f}}$. This is discussed in general in Sec. II, whereas particular examples are considered in the subsequent sections.

\section{THERMODYNAMICAL VOLUME AND PRESSURE OF A SOLITON}

In [4] it was proven that $V_{\mathrm{g}}=V_{\mathrm{t}} \Rightarrow P_{\mathrm{t}}=P_{\mathrm{f}}$ for scalar field theories with zero-dimensional vacuum manifolds in flat space $\mathbb{R}^{d}$. Here we generalize these results to more general field theories (e.g., gauge theories, like the AbelianHiggs model, which supports vortices with a vacuum manifold $\mathbb{S}^{1}$ ) and to more general space manifolds. For this purpose, let us consider the following constrained energy functional

$E_{\mathrm{c}}=E+C_{\mathrm{vol}}, \quad C_{\mathrm{vol}}=P\left(\int d^{d} x \sqrt{g} \Theta(\mathcal{E}(x))-V\right)$.

Here, $C_{\mathrm{vol}}$ is the volume constraint imposing the condition $V=V_{\mathrm{g}}$, and $P$ is the corresponding Lagrange multiplier. Further, the generalized step function $\Theta(\mathcal{E}(x))$ is the locus function of a field configuration, i.e.,

$$
\Theta(\mathcal{E}(x))= \begin{cases}1 & \text { for } \mathcal{E}(x)>0 \\ 0 & \text { for } \mathcal{E}(x)=0,\end{cases}
$$

which, obviously, implies $\int d^{d} x \sqrt{g} \Theta(\mathcal{E}(x))=V_{\mathrm{g}}$. The explicit expression for the locus function chosen here is different from the one in [4] (although they coincide for the cases considered in [4]) and the expression used here allows us to consider more general cases. The important point for us is that the locus function is invariant with respect to infinitesimal variations of the metric (the relevance of this condition will become clear in a moment). This invariance follows from the following observations. Some terms in $\mathcal{E}$ (e.g., potentials) do not depend on the metric, at all, so invariance is obvious. The metric dependent terms in $\mathcal{E}$ may always be written as nondegenerate quadratic forms $v^{a} M^{a b} v^{b}$ where $M^{a b}$ is a positive definite matrix constructed from the metric, whereas the $v^{a}$ are constructed from the fields and their (partial or gauge-covariant) derivatives. Examples are the standard (gauge) kinetic term where $M^{a b} \rightarrow g^{i j}$ and $v^{a} \rightarrow \partial_{i} \phi$ [or $v^{a} \rightarrow D_{i} \phi=\left(\partial_{i}-i e A_{i}\right) \phi$ for a complex $\phi$ coupled to a gauge field], or Skyrme-type terms, where $M^{a b} \rightarrow g^{i j} g^{k l} \quad$ and $v^{a} \rightarrow \partial_{i} \phi^{1} \partial_{k} \phi^{2}-$ $\partial_{i} \phi^{2} \partial_{k} \phi^{1}$ for two fields $\phi^{1}, \phi^{2}$. The important point is that all these expressions are 0 if and only if $v^{a}=0$ (a vacuum configuration), independently of the metric, because of the positive definite nature of $M^{a b}$. It follows immediately that the locus function is invariant under variations of the metric.

The Lagrange multiplier $P$ obeys the thermodynamical relation $P=-\left(d E_{\mathrm{c}} / d V\right)$, by construction. We now want to prove that it is, at the same time, equal to the fieldtheoretical pressure (1.4). In a first step, we prove it for flat space $g_{i j}=\delta_{i j}, g=1$, closely following [4]. We act with an infinitesimal scale transformation $x^{i} \rightarrow(1+\lambda) x^{i}$, for infinitesimal $\lambda$, on all fields that appear in the constrained energy functional (but not on the metric). Then we assume that all terms in the energy density are "geometrically natural" [14] such that a coordinate transformation acting on the fields may be traded for the inverse coordinate transformation of the metric, $\delta_{i j} \rightarrow(1-2 \lambda) \delta_{i j}$. That is to say, we act with the transformation

$$
\delta=\left.\int d^{d} x \delta g_{i j}(\vec{x}) \frac{\delta}{\delta g_{i j}(\vec{x})}\right|_{g_{i j}=-\delta_{i j}}
$$

where $\delta g_{i j}=-2 \lambda g_{i j}$ on $E_{\mathrm{c}}$. Variation of $E$ just gives

$$
\delta E=\lambda \int d^{d} x \sqrt{g} g_{i j} T^{i j}=\lambda d \int d^{d} x \sqrt{g} \mathcal{P}=\lambda d V P_{\mathrm{f}}
$$

whereas variation of $C_{\mathrm{vol}}$ gives

$$
\delta C_{\mathrm{vol}}=-P \lambda d \int d^{d} x \sqrt{g} \Theta(\mathcal{E})=-P \lambda d V
$$

where we used the invariance of $\Theta(\mathcal{E})$. Further, we wrote the expressions for general $g_{i j}$, for later convenience. To close the argument, we remember that the variation $\delta$ came from a variation of the fields (a scale transformation). But for solutions of the variational problem (2.1), the functional is invariant under any field variation, that is, on shell (for solutions) $\delta E_{\mathrm{c}}=0$, immediately leading to $P=P_{\mathrm{f}}$, which is what we wanted to prove.

The question is whether this argument can be generalized for general space manifolds $\mathcal{M}$. After all, in Eqs. (2.4) and (2.5) only the constant Weyl transformation $g_{i j} \rightarrow \mathrm{e}^{-2 \lambda} g_{i j}$ appears, which can be defined for any metric. The apparent problem is that, as this Weyl transformation no longer follows from a variation of the fields (i.e., from a coordinate 
transformation), there is no obvious reason why $\delta E_{\mathrm{c}}=0$ should hold on shell. Nevertheless, if we accept (i.e., define) that the volume of a soliton is varied via a Weyl transformation and not by squeezing the soliton for a fixed metric (on a fixed background manifold), then the result $P_{\mathrm{t}}=P_{\mathrm{f}}$ follows immediately and does not even require the introduction of the constrained energy functional $E_{\mathrm{c}}$. The reason is that in this case the operations of a constant Weyl transformation acting on $E$ (which defines $P_{\mathrm{f}}$ ) and of a variation of the volume (which defines $P_{\mathrm{t}}$ ) are identical.

Squeezing a soliton (i.e., to introduce pressure) via a Weyl rescaling (i.e., by squeezing the whole manifold) may appear counterintuitive in a first instant, but here we argue that it is a natural definition in the general case. First of all, on $\mathbb{R}^{d}$ it is equivalent to the standard idea of squeezing the soliton itself. Secondly, for general $g_{i j}$ there are infinitely many possibilities to squeeze a soliton on a fixed background manifold, and there is no preferred "symmetric" or "shape-preserving" way of squeezing-unless the metric itself has some symmetries (Killing vectors). Squeezing via a constant Weyl rescaling, on the other hand, is shape preserving by construction and, therefore, the most symmetric definition to squeeze a soliton, i.e., to change its volume. In addition, if a soliton on a compact manifold covers the whole manifold (i.e., $V=V_{\mathcal{M}}$ ), it is not clear how to squeeze the soliton to a smaller volume for a fixed background without drastically changing its boundary conditions. We remark that the Weyl rescaling definition for the variation of the volume of a soliton implies that the pressure of a BPS soliton is always 0 . Indeed, the energy of a BPS soliton is proportional to a topological invariant (winding number etc.) that is metric independent by definition. It follows that the Weyl variation of the onshell energy and, therefore, the pressure, is identically 0 .

\section{THE BRADLOW BOUND-ABELIAN-HIGGS VORTICES}

\section{A. The Abelian-Higgs vortices at critical coupling}

After having established that the geometric volume is, in fact, the thermodynamic volume, we investigate its relation with the Bradlow volume $V_{B}$ and the volume of the space manifold $V_{\mathcal{M}}$. Furthermore, we show that the Bradlow volume may be used as a rather universal characteristic of general BPS-type theories.

Let us briefly demonstrate the original derivation of the Bradlow bound for solitons in the Abelian-Higgs model at critical coupling on an arbitrary compact manifold $\mathcal{M}$ $[10,15]$,

$L=\int_{\mathcal{M}}\left(-\frac{1}{4} F_{\mu \nu}^{2}+\frac{1}{2} \overline{D_{\mu} u} D^{\mu} u-\frac{1}{8}(1-u \bar{u})^{2}\right) \Omega d^{2} x$,

where $D_{\mu} u=\left(\partial_{\mu}-i A_{\mu}\right) u$, and the metric is

$$
d s^{2}=d t^{2}-\Omega\left(x^{1}, x^{2}\right)\left(\left(d x^{1}\right)^{2}+\left(d x^{2}\right)^{2}\right) .
$$

The energy bound is

$$
E \geq \pi N
$$

where $N$ is the topological charge, and the static energy is

$$
\begin{aligned}
E= & \frac{1}{2} \int_{\mathcal{M}}\left(\Omega^{-1} B^{2}+\overline{D_{1} u} D_{1} u+\overline{D_{2} u} D_{2} u\right. \\
& \left.+\frac{\Omega}{4}(1-u \bar{u})^{2}\right) d^{2} x .
\end{aligned}
$$

The bound is saturated for configurations obeying the Bogomolny equations

$$
\begin{gathered}
D_{1} u+i D_{2} u=0, \\
B-\frac{\Omega}{2}(1-u \bar{u})=0 .
\end{gathered}
$$

To get the Bradlow bound, we integrate the last equation over the physical (base) space manifold $\mathcal{M}$. Then,

$$
2 \int_{\mathcal{M}} B d^{2} x+\int_{\mathcal{M}} u \bar{u} \Omega d^{2} x=\int_{\mathcal{M}} \Omega d^{2} x
$$

If the base space is a compact surface without boundary, the first Chern number must be an integer $N$, i.e., the magnetic flux $\Phi_{B} \equiv \int d^{2} x B=2 \pi N$, so

$$
4 \pi N+\int_{\mathcal{M}} u \bar{u} \Omega d^{2} x=V_{\mathcal{M}},
$$

where $V_{\mathcal{M}}=\int_{\mathcal{M}} \Omega d^{2} x$ is the total area (two-dimensional volume) of the physical space. Hence, finally, we arrive at the bound

$$
V_{\mathcal{M}} \geq 4 \pi N .
$$

The meaning of the bound is the following. For a given value of the topological charge $N$, solutions of the Bogomolny equations (BPS vortices) do not exist unless the area of the (compact) base space is bigger than $4 \pi N$. For a fixed volume of the base-space manifold, one can put a finite number of BPS vortices up to a maximal number (provided by the bound), $N \leq\left(V_{\mathcal{M}} / 4 \pi\right)$, above which vortices are no longer solutions of the Bogomolny equations but, instead, solve the full second-order equations of motion. Here we introduce the notion of the Bradlow volume $V_{B}$ as the minimal volume of the manifold $\mathcal{M}$ for which BPS vortices (of a given topological charge) do exist. Obviously, this quantity can be defined for any model with BPS solitons.

Definition.-The Bradlow volume $V_{B}$ is the minimal volume of the base-space (compact) manifold $\mathcal{M}$ for which 
the BPS sector of a solitonic model has a nontrivial solution.

The energy of the BPS vortices $\left(V_{\mathcal{M}} \geq V_{B}\right)$ is independent of the base-space volume and takes the constant value

$$
E=\pi N .
$$

Below the bound, for $V_{\mathcal{M}}<V_{B}$, there only exists the "constant" non-BPS solution of the full second-order EulerLagrange (EL) equations

$$
u=0, \quad B=2 \pi N V^{-1} \Omega
$$

with energy

$$
E[V]=\frac{(2 \pi N)^{2}}{2 V}+\frac{V}{8} .
$$

In all cases, the geometric (thermodynamical) volume $V$ of a vortex is equal to $V_{\mathcal{M}}$, as the vortices cover the full base space. Then we define pressure as

$$
P=-\left(\frac{\partial E}{\partial V}\right) .
$$

For BPS solutions, the energy $E=\pi N$ is independent of the volume; hence $P=0$ for $V \geq V_{B}$. Note that also the pressure density is identically 0 in this case due to the Bogomolny equations. Below the bound $\left(V \leq V_{B}\right)$, a nontrivial positive pressure appears,

$$
P=\frac{(2 \pi N)^{2}}{2 V^{2}}-\frac{1}{8}=\frac{1}{8}\left(\frac{V_{B}^{2}}{V^{2}}-1\right) .
$$

As a consequence, one could say that the Abelian-Higgs model on a compact manifold at critical coupling and large (topological charge or energy) density describes solitonic matter with a maximally stiff mean-field equation of state. In fact, the mean-field energy density reads

$$
\bar{\epsilon}=\frac{E}{V}=\frac{(2 \pi N)^{2}}{2 V^{2}}+\frac{1}{8}=\frac{1}{8}\left(\frac{V_{B}^{2}}{V^{2}}+1\right) .
$$

Hence,

$$
\bar{\epsilon}=P+\frac{1}{4} .
$$

This equation of state for vortices is valid for the case of a compact manifold $\mathcal{M}$ without boundary. This means that it does not have to be identical to the equation of state of vortex matter in the plane, which represents a system of vortices enclosed in a finite volume subspace of the original $\mathbb{R}^{2}$ space. In such a situation, that is a vortex in a finite flat disc $\mathbb{D} \subset \mathbb{R}^{2}$, one has to carefully face the issue of the boundary conditions. On $\mathbb{R}^{2}$, the first Chern number does not have to be an integer, and the quantization of the magnetic flux is, instead, a consequence of the condition of finite energy. In polar coordinates $\mu=t, r, \varphi$, finite static energy requires $\lim _{r \rightarrow \infty} u=\mathrm{e}^{i \alpha(\varphi)}$ and, in the gauge $A_{t}=0$, $A_{r}=0, \lim _{r \rightarrow \infty} A_{\varphi}=\partial_{\varphi} \alpha$, and the vortex number is given by the winding of $\alpha$ at infinity, $\alpha(2 \pi)-\alpha(0)=2 \pi N$.

On a finite disc $\mathbb{D} \subset \mathbb{R}^{2}$, there exist two possibilities to put a vortex with vorticity $N$ such that $u$ at the boundary of $\mathbb{D}$ still is $\left.u\right|_{\partial \mathbb{D}}=\mathrm{e}^{i \alpha}$ and $\alpha$ changes by $2 \pi N$ while traversing the boundary once (i.e., imposing Dirichlet boundary conditions on the field $u$ ). The first possibility consists in requiring that the vortex still obeys the BPS equations. It is then no longer possible to require that the gauge field approaches a pure gauge at the boundary and, consequently, the magnetic flux is no longer quantized [16]. The easiest way to see this is by assuming a round disc with radius $R$ and the spherically symmetric ansatz

$u(r, \varphi)=\mathrm{e}^{i N \varphi} f(r), \quad A_{\varphi}=N-a(r) \Rightarrow B=-\frac{a^{\prime}}{r}$

leading to the BPS equations

$$
f^{\prime}=\frac{a f}{r}, \quad a^{\prime}=-\frac{1}{2} r\left(1-f^{2}\right) .
$$

On $\mathbb{R}^{2}$, the boundary conditions for a vortex are $f(0)=0$, $a(0)=N$ and $\lim _{r \rightarrow \infty} f(r)=1, \lim _{r \rightarrow \infty} a(r)=0$. On a finite disc, the two conditions $f(R)=1$ and $a(R)=0$ together, however, are too strong (as may be checked easily) and only permit the trivial solution $f=1, a=0$. Requiring $f(R)=1$ (Dirichlet boundary condition) therefore forces us to allow for a nonzero $a(R) \equiv a_{R}$, and the magnetic flux $\Phi_{B}=2 \pi \int d r r B=2 \pi\left(N-a_{R}\right)$ is no longer quantized. The energy, on the other hand, still takes the BPS value $E=\pi N$, and the pressure is, therefore, 0 . These solutions exist on discs of arbitrary size [16]; therefore, there is no Bradlow bound for the Abelian-Higgs model at the critical coupling on a flat finite disc $\mathbb{D}$. Both the fact that the pressure remains 0 and that the magnetic flux changes its value with the disc size implies that these vortices should not be interpreted as squeezed versions of the original vortex on $\mathbb{R}^{2}$.

The second possibility consists in requiring both that $\left.u\right|_{\partial \mathbb{D}}=\mathrm{e}^{i \alpha}$ and that $A_{\mu}$ is pure gauge at the boundary of the disc, such that the magnetic flux remains quantized. The corresponding vortex can no longer obey the BPS equations, but it may still solve the full (static) second-order Euler-Lagrange equations. In particular, for a spherically symmetric disc and for the spherically symmetric ansatz from above, we may now impose $f(R)=1$ and $a(R)=0$. The resulting solution has a quantized magnetic flux and a nonzero pressure (because it is not BPS) and may, 
therefore, now be interpreted as a squeezed version of the spherically symmetric BPS vortex on $\mathbb{R}^{2}$.

We remark that in the recent paper [17] several versions of vortex equations related to but different from the standard BPS equation (3.6) have been considered, leading to interesting variations of the Bradlow bound. In one case, a BPS vortex equation with an "inverse" Bradlow bound $N \geq\left(V_{\mathcal{M}} / 4 \pi\right)$ has been found, where the vortex number must be above a certain minimum value. The BPS vortices in this model are, however, critical points but not minima of the energy functional, which is not even bound from below. The corresponding vortex equation itself was already introduced in [18] in a different context. Another BPS vortex equation studied in [17] ("Bradlow vortices") may have solutions only if the volume of the manifold is equal to the Bradlow volume, so that the "Bradlow bound" is converted into a "Bradlow equation" (for more on this model see [19]).

\section{B. Generalized Abelian-Higgs vortices}

It has been observed recently [20] that one can generalize the Abelian-Higgs model preserving its BPS property by allowing for two field dependent non-negative coupling functions that multiply the gauge and the Higgs kinetic parts of the action,

$L_{\mathrm{gen}}=\int\left(-\frac{G\left(|u|^{2}\right)}{4} F_{\mu \nu}^{2}+\frac{w\left(|u|^{2}\right)}{2} \overline{D_{\mu} u} D^{\mu} u-U\right) \Omega d^{2} x$.

Here, the potential $U(u \bar{u})$ is always assumed to take its vacuum value at $|u|=1$, i.e., $U(1)=0$. Further, $w$ is related to a nontrivial target space metric, whereas $G$ defines a kind of generalized magnetic permeability (we remark that the case $G=1, w \neq 1$ was already considered in [21]). The model has a BPS sector if the coupling functions $G, w$ and the potential $U$ obey $(f \equiv|u|)$

$$
\frac{d}{d f} \sqrt{G U}=\frac{1}{\sqrt{2}} w f
$$

or, for the simplifying assumption $w=1$,

$$
G U=\frac{1}{8}\left(1-f^{2}\right)^{2} .
$$

The Bogomolny equations in this case are [20,22]

$$
\begin{gathered}
D_{1} u+i D_{2} u=0 \\
B-\frac{\Omega}{2} \frac{(1-u \bar{u})}{G(u \bar{u})} \equiv B-4 \Omega \frac{U(u \bar{u})}{1-u \bar{u}}=0,
\end{gathered}
$$

and the corresponding BPS energy is still $E_{\mathrm{BPS}}=\pi N$. The question is whether for these models, when defined on a compact space manifold $\mathcal{M}$ without boundary, there exists a Bradlow bound $V_{B}$ such that the above BPS equations may be solved for $V_{\mathcal{M}} \geq V_{B}$. Here we give some indications that this may be the case, at least for some choices of $G$. First of all, the above BPS equations have the constant, trivial solution

$$
u=0, \quad B=\frac{\Omega}{2 G(0)} .
$$

As a consequence of the magnetic flux quantization, the manifold where this solution exists must have the Bradlow volume

$$
V_{B}=4 \pi N G(0)=\frac{\pi N}{2 U(0)} .
$$

Next we observe that, for arbitrary volume $V_{\mathcal{M}}$, the full second-order EL equations have the constant solution

$$
u=0, \quad B=2 \pi N V_{\mathcal{M}}^{-1} \Omega .
$$

The corresponding static energy is

$$
\begin{aligned}
E & =\frac{1}{2} \int d^{2} x\left(\frac{G(0)}{\Omega} B^{2}+2 \Omega U(0)\right) \\
& =(2 \pi N)^{2} \frac{G(0)}{2 V}+U(0) V
\end{aligned}
$$

(where $V=V_{\mathcal{M}}$ ). Further, this energy takes its minimal value $E=E_{\mathrm{BPS}}=\pi N$ exactly at $V_{\mathcal{M}}=V_{B}=4 \pi N G(0)$ [where we used that $U(0)=(8 G(0))^{-1}$ ] and leads to positive pressure for $V_{\mathcal{M}}<V_{B}$ but to negative pressure for $V_{\mathcal{M}}>V_{B}$. This indicates an instability of the formal solution (3.26) for $V_{\mathcal{M}}>V_{B}$, i.e., the existence of field configurations with lower energy. A different question is whether local extrema (static solutions) or global minima (BPS solutions) of the energy functional may be found among these low-energy configurations. A general answer to this question for general $G$ is beyond the scope of the present paper. We also find some indications that the answer to this question may be quite sensitive to the choice of the coupling function $G$. For let us assume that BPS solutions exist for $V_{\mathcal{M}} \geq V_{B}$ and provide the true, stable solutions. A first, related question to ask is under which circumstances the Bradlow volume (3.25) (which has a trivial solution with BPS energy) provides, at the same time, a Bradlow bound (such that BPS solutions can exist only for $V_{\mathcal{M}} \geq V_{B}$, but not for $\left.V_{\mathcal{M}}<V_{B}\right)$. Integrating the second BPS equation over the manifold, the resulting equation may be expressed as

$$
V_{B}=V_{\mathcal{M}}-\frac{1}{U(0)} \int d^{2} x \Omega\left(U(0)-\frac{U(u \bar{u})}{1-u \bar{u}}\right) .
$$


This implies $V_{B} \leq V_{\mathcal{M}}$ provided that the second term on the rhs is non-negative. A sufficient condition for this is

$$
U(u \bar{u}) \leq U(0)(1-u \bar{u}) .
$$

This condition is also necessary in a certain sense, because otherwise the condition $V_{B} \leq V_{\mathcal{M}}$ would not be universal and would depend on the field configuration $u$ (it would be broken by fields that take such values in most of $\mathcal{M}$ that violate the above inequality) and on the volume element $\Omega$ $(\Omega$ could enhance the contribution of these "forbidden" regions). A class of potentials that obey (3.29) is

$$
U=(1-u \bar{u})^{\alpha}, \quad \alpha \geq 1,
$$

corresponding to $G=(1 / 8)(1-u \bar{u})^{2-\alpha}$.

Now, let us assume that we chose a potential obeying (3.29) and, further, let us assume that BPS vortex solutions exist for $V_{\mathcal{M}}>V_{B}$. Then these BPS vortex solutions may show two rather different types of behavior for $V_{\mathcal{M}}$ close to $V_{B}$. The first possibility is that the BPS vortex solution approaches the constant solution (3.24) in the limit $V_{\mathcal{M}} \rightarrow V_{B}$. In other words, the stable constant solution (3.26) for $V_{\mathcal{M}}<V_{B}$ bifurcates at $V_{\mathcal{M}}=V_{B}$ into an unstable constant solution and a stable BPS solution. This is known to happen, e.g., for the standard AbelianHiggs model at critical coupling $\left[U=(1 / 8)(1-u \bar{u})^{2}\right]$. It is plausible to conjecture that this behavior occurs for deformation functions $G$ that do not deviate too much from the standard Abelian-Higgs case $G=1$. In physical terms, one might say that at $V_{\mathcal{M}}=V_{B}$ a second-order phase transition occurs. The second possibility is that the BPS vortex solution is completely different from the constant solution (3.24) even for $V_{\mathcal{M}}$ very close to $V_{B}$, despite the fact that they have the same energy. In the limit $V_{\mathcal{M}} \rightarrow V_{B}$ the BPS vortex may either approach a solution different from (3.24), or the limit is not attainable, i.e., a limiting solution does not exist. In both cases, the phase transition is of first order. This possibility of a first-order phase transition may appear strange at first sight, but we find that it exists and is realized by compact BPS vortex solutions, i.e., BPS vortices that deviate from their vacuum value only in a finite region with the topology of a disc. Two particular cases of compact vortices allowing for explicit solutions are considered in the next subsection.

We end this section by remarking that there exists the particular case $G=|u|$, which allows us to reduce the BPS equations on $\mathbb{R}^{2}$ to the (integrable) elliptic sinh-Gordon equation for $g=\ln |u|$. It can be demonstrated that in this case a vortex solution exists for $N=1$ but not for higher vortex number [23], although an explicit expression for this solution of the elliptic sinh-Gordon equation (obeying the boundary conditions of a vortex) cannot be found.

\section{Compact generalized Abelian-Higgs vortices}

Very recently, some examples of compact BPS vortices, i.e., BPS vortices that deviate from their vacuum value only in a finite subregion (the "locus set" of the vortex) of $\mathbb{R}^{2}$, have been constructed for the generalized Abelian-Higgs model in [24]. This implies that these compact vortices continue to exist on space manifolds that are themselves finite subsets of $\mathbb{R}^{2}$ (discs), provided that the locus set of the compact vortex is completely contained within the disc. A different question is whether the corresponding models may support BPS vortices on compact manifolds without boundary. We find that the characteristic behavior of such solutions depends on the specific model under consideration; therefore, we just consider two particular examples found in [24]. We remark that the compacton solutions in [24] were constructed as limiting cases of regular, noncompact vortices, demonstrating that compactons (with low regularity at the compacton boundary) may be understood as limiting cases of noncompact vortices with high regularity (infinitely differentiable).

\section{Case $G=\left.|1-| u\right|^{2} \mid$}

For $G=\left|1-f^{2}\right|, f \equiv|u|$, (and $w=1$ ), the resulting potential reads

$$
U=\frac{1}{8}\left|1-f^{2}\right| .
$$

This potential approaches the vacuum at $f=1$ less than quadratically (in fact, linearly) and, therefore, leads to compacton solutions, which are of low regularity at the compacton boundary. Using the spherically symmetric ansatz (3.17) in $\mathbb{R}^{2}$, the resulting BPS equations in the fundamental domain of the vortex (for $0 \leq f \leq 1$ ) are

$$
f^{\prime}=\frac{a f}{r}, \quad a^{\prime}=-\frac{r}{2} .
$$

The second equation has the solution

$$
a= \begin{cases}N-\frac{r^{2}}{4} & r \leq 2 \sqrt{N} \\ 0 & r>2 \sqrt{N}\end{cases}
$$

and the compacton radius is $r_{c}=2 \sqrt{N}$, leading to the compacton volume $V_{c}=\pi r_{c}^{2}=4 \pi N$, which exactly agrees with the Bradlow volume $V_{B}=4 \pi N G(0)=4 \pi N$. The gauge potential $a$ is continuous at the compacton boundary, but the resulting magnetic field

$$
B= \begin{cases}\frac{1}{2} & r \leq 2 \sqrt{N} \\ 0 & r>2 \sqrt{N}\end{cases}
$$

is discontinuous. Finally, integration of the first BPS equation leads to 


$$
f= \begin{cases}\left(\frac{r}{2 \sqrt{N}}\right)^{N} \mathrm{e}^{\frac{2 N-r^{2}}{8}} & r \leq 2 \sqrt{N} \\ 1 & r>2 \sqrt{N}\end{cases}
$$

Both $f$ and $f^{\prime}$ are continuous at $r=r_{c}=2 \sqrt{N}$.

The fact that the Bradlow volume and the compacton volume agree makes one suspect that compact BPS vortices continue to exist on compact manifolds $\mathcal{M}$ without boundary provided that the volume of the manifold is sufficiently big, $V_{\mathcal{M}}>V_{B}$. Here we demonstrate that this is indeed true for the specific case $\mathcal{M}=\mathbb{S}^{2}$. For our purposes, it is useful to generalize the two BPS equations (3.22) and (3.23) [which, as they stand, only hold for metrics of the form (3.2)] to space metrics on $\mathcal{M}$ in arbitrary coordinates, i.e., $d s_{\mathcal{M}}^{2}=g_{i j} d x^{i} d x^{j}$. For the second BPS equation this is easy, because it may immediately be written as an equation between two-forms,

$$
\mathbf{B}=\frac{1}{2} \mathbf{v o l},
$$

where $\mathbf{B}=d \mathbf{A}$ is the magnetic two-form, and vol is the volume form (area two-form) on $\mathcal{M}$. In particular, for the metric on $\mathbb{S}^{2}$ in longitude and latitude coordinates,

$$
d s_{\mathbb{S}^{2}}^{2}=R^{2}\left(d \theta^{2}+\sin ^{2} \theta d \varphi^{2}\right)
$$

(where $R$ is the-dimensionless-radius of the sphere), and for the rotationally symmetric ansatz

$$
\mathbf{A}=(N-a(\theta)) d \varphi, \quad u=f(\theta) \mathrm{e}^{i N \varphi},
$$

this equation leads to

$$
a_{, \theta}=-\frac{R^{2}}{2} \sin \theta
$$

and the formal solution obeying the boundary condition $a(\theta=0)=N$ is

$$
a=\frac{R^{2}}{2}(\cos \theta-1)+N .
$$

This solution may be extended to a compacton solution

$$
a=\left\{\begin{array}{ll}
\frac{R^{2}}{2}(\cos \theta-1)+N & \theta \leq \theta_{c} \\
0 & \theta>\theta_{c},
\end{array} \quad \cos \theta_{c}=\frac{R^{2}-2 N}{R^{2}}\right.
$$

provided that $R^{2}>N$, that is, $V_{\mathbb{S}^{2}} \equiv 4 \pi R^{2}>4 \pi N \equiv V_{B}$. So, as expected, the compacton solutions on $\mathbb{S}^{2}$ exist if the area of the two sphere exceeds the Bradlow volume (area) $V_{B}$.
The first BPS equation (3.22) may be expressed in a coordinate-independent way as follows. Temporarily renaming the coordinates like $x^{1} \rightarrow x, x^{2} \rightarrow y$, (3.22) reads $D_{y} u=i D_{x} u$ or, after introducing the complex-valued oneform

$$
\mathrm{D} u=D_{x} u d x+D_{y} u d y,
$$

$\mathrm{D} u\left(\partial_{y}\right)=i \mathrm{D} u\left(\partial_{x}\right)$. In other words, evaluating $\mathrm{D} u$ on the vector rotated by 90 degrees in the counterclockwise sense $\partial_{x} \rightarrow \partial_{y}$ is equivalent to multiplying $\mathrm{D} u\left(\partial_{x}\right)$ by $i$. But this has a simple coordinate-invariant generalization on any oriented Riemannian two manifold $\mathcal{M}$. Then $\mathrm{D} u$ is a linear map from the tangent space to the complex numbers, $\mathrm{D} u: T_{p} \mathcal{M} \rightarrow \mathbb{C}$. We may define the following complex structures on the two vector spaces $\mathbb{C}$ and $T_{p} \mathcal{M}$. On $\mathbb{C}$ it is just multiplication by $i$, and on $T_{p} \mathcal{M}$, rotation by 90 degrees counterclockwise, as determined by the metric and orientation on $\mathcal{M}$. Then the first BPS equation in its coordinate-invariant form just says that $\mathrm{D} u$ is complex linear, that is,

$$
\mathrm{D} u(J X)=i \mathrm{D} u(X)
$$

for arbitrary vectors $X$. In particular, for the metric (3.37) and for the vector $X=\partial_{\theta} \Rightarrow J X=(1 / \sin \theta) \partial_{\varphi}$, this equation reads $(1 / \sin \theta) D_{\varphi} u=i D_{\theta} u$, which, for the ansatz (3.38), leads to the first-order ordinary differential equation (ODE)

$$
f_{, \theta}=\frac{a f}{\sin \theta} .
$$

The solution may be found easily by introducing the new variable $t=\cos \theta$ and, with the correct choice of normalization, reads

$$
f= \begin{cases}\left(\frac{\cos \theta+1}{\cos \theta_{c}+1}\right)^{\frac{R^{2}-N}{2}}\left(\frac{1-\cos \theta}{1-\cos \theta_{c}}\right)^{\frac{N}{2}} & \theta \leq \theta_{c} \\ 1 & \theta>\theta_{c} .\end{cases}
$$

It may be checked that $f\left(\theta_{c}\right)=1$, and $f^{\prime}\left(\theta_{c}\right)=0$.

Obviously, the BPS compacton solution that covers the whole fundamental domain $0 \leq f \leq 1$ is completely different from the constant solution (3.26) even for a $V_{\mathbb{S}^{2}}$ very close to $V_{B}$. The phase transition from the constant solution to the BPS solution is, therefore, a first-order transition in the case of compactons. Further, for compact BPS vortices the limit $V_{\mathcal{M}} \rightarrow V_{B}$ is not attainable. In this limit, the boundary of the compacton (with the topology of $\mathbb{S}^{1}$ ) has to shrink to a point, which is incompatible with the nontrivial boundary condition for the Higgs field $u$ (it must wind $N$ times about the boundary for vortex number $N$ ). 


\section{Case $\boldsymbol{G}=\left.\left.(2 / 3)|\boldsymbol{1}-| \boldsymbol{u}\right|^{2}|/| \boldsymbol{u}\right|^{2}$}

First of all, for $G=(2 / 3)\left|1-f^{2}\right| / f^{2}$ we observe that the Bradlow volume $V_{B}=4 \pi N G(0)=\infty$ is infinite. A related fact is that the constant solution (3.26) does not exist (i.e., has infinite energy). Also, the corresponding potential does not obey the inequality (3.29). Unsurprisingly, thus, the solutions on $\mathbb{R}^{2}$ found in [24] behave in a way that is quite different from the case considered in the previous subsection. BPS solutions are still compact vortices, but the compacton radius now is $r_{c}=\sqrt{N(N+2) / 3}$, leading to a compacton volume $V_{c}=\pi r_{c}^{2}=(\pi / 3) N(N+2)$ that grows faster than linear (approximately quadratically) with the winding number. It is plausible to conjecture that there exists a relation between this unusual growth of the compacton volume, on the one hand, and the nonexistence of a (finite) Bradlow volume, on the other hand, because any (finite) Bradlow volume proportional to $N$ would be surpassed by this compacton volume for sufficiently large $N$. On the other hand, compact BPS vortices continue to exist at least on some compact manifolds without boundary provided their volume is sufficiently large. Indeed, from any compact vortex solution on $\mathbb{R}^{2}$ we immediately get a solution on a sufficiently large disc $\mathbb{D} \subset \mathbb{R}^{2}$ with the flat metric. From there we get a solution on a compact manifold with the topology of a two sphere by just gluing this disc to a second disc along the boundaries of the two discs. The first disc may even be curved in the region outside the compacton. The gauge potential cannot be well defined on the whole second disc (it is a large gauge transformation there), but this problem may be remedied by acting with the inverse of this large gauge transformation on $A_{\mu}$ and on $u$. It is well known that on a compact manifold without boundary, in any case, one gauge potential cannot be defined globally and, instead, several potentials have to be chosen on several patches, related by large gauge transformations in the overlap regions (it is this fact that gives rise to the quantization of the magnetic flux in the first place). Solutions on more complicated manifolds may be constructed by gluing the first disc to more complicated surfaces with $\mathbb{S}^{1}$ boundaries instead of the second disc. A different question is whether solutions exist on manifolds where the metric deviates from the flat one in the region of the compacton. This problem is, however, beyond the scope of the present paper.

We conclude that, in this and related cases, a Bradlow bound as a useful criterion for the existence of BPS vortex solutions does not seem to exist (the formal Bradlow volume is infinite, but BPS solutions on manifolds with finite volumes still exist). We could, of course, define the compacton volume for each $N$ as the Bradlow volume, but this is not a useful definition because (i) it is not given a priori, i.e., we have to find the compact BPS solution and its volume to get the Bradlow volume and, (ii) there is no guarantee that the Bradlow volume will be independent of the space manifold $\mathcal{M}$ (BPS solutions on different $\mathcal{M}$ might have different volumes).

\section{PERFECT FLUID FIELD THEORIES}

Another class of models for which we investigate the relation between the (geometric/thermodynamic) volume and the Bradlow volume is a family of models describing perfect fluids. This means that the corresponding action leads to an energy-momentum tensor of the perfect fluid type

$$
T^{\mu \nu}=(p+\rho) u^{\mu} u^{\nu}-p g^{\mu \nu},
$$

where $p$ is the pressure, $\rho$ the proper energy density and $u^{\mu}$ the four-velocity.

\section{A. Scalar field in $1+1$ dimensions}

The simplest example of a perfect fluid type action is provided by a real scalar field in $(1+1)$ dimensions

$$
\mathcal{L}=\frac{1}{2} \partial_{\mu} \phi \partial^{\mu} \phi-m^{2} U(\phi)
$$

Then the energy-momentum tensor reads

$$
T_{\mu \nu}=\partial_{\mu} \phi \partial_{\nu} \phi-g_{\mu \nu} \mathcal{L}=u_{\mu} u_{\nu}(\mathcal{E}+\mathcal{P})-g_{\mu \nu} \mathcal{P}
$$

where the proper energy density and pressure density are

$$
\begin{aligned}
& \mathcal{E}=-\frac{1}{2} \partial_{\mu} \phi \partial^{\mu} \phi+m^{2} U(\phi), \\
& \mathcal{P}=-\frac{1}{2} \partial_{\mu} \phi \partial^{\mu} \phi-m^{2} U(\phi),
\end{aligned}
$$

while the "two-velocity" $u_{\mu}=\left(-\partial_{\nu} \phi \partial^{\nu} \phi\right)^{-1 / 2} \epsilon_{\mu \rho} \partial^{\rho} \phi$. For static configurations, the proper energy density and pressure density are simply components of the energymomentum tensor

$$
\begin{aligned}
& \mathcal{E}=T_{00}=\frac{1}{2} \phi^{\prime 2}+m^{2} U(\phi), \\
& \mathcal{P}=T_{11}=\frac{1}{2} \phi^{\prime 2}-m^{2} U(\phi) .
\end{aligned}
$$

Furthermore, due to the conservation of the energymomentum tensor the pressure density has to take a constant value $\mathcal{P}=P$, which is simply an integration constant when we integrate the full second-order static field equation once. Now we assume that the potential has at least two vacua at $\phi_{+}$and $\phi_{-}$, which leads to the emergence of kinks (topological solitons) as configurations interpolating between them. Here the topological current is 


$$
j_{\mu}=-\frac{1}{\phi_{+}-\phi_{-}} \epsilon_{\mu \nu} \partial^{\nu} \phi .
$$

The energy of a kink is bounded from below by a topological bound

$$
\begin{aligned}
E & =\frac{1}{2} \int_{-\infty}^{\infty} d x\left(\phi^{\prime 2}+2 m^{2} U\right) \geq\left|m \int d x \sqrt{2 U} \phi^{\prime}\right| \\
& =m\left|\int_{\phi_{-}}^{\phi_{+}} d \phi \sqrt{2 U}\right|,
\end{aligned}
$$

where the last integral is over the "fundamental domain" of target space (the region $\phi_{-} \leq \phi \leq \phi_{+}$where the soliton takes its values). The bound is saturated by solutions of the zero-pressure equation called the BPS equation

$$
\frac{1}{2} \phi^{\prime 2}-m^{2} U(\phi)=0 .
$$

Such BPS kinks are global energy minima in the respective topological sectors. However, kink solutions exist also for any positive value of the pressure. Their energy is again given by a target space (solution independent) formula

$$
E(P)=\frac{1}{\sqrt{2}} \int_{\phi_{-}}^{\phi_{+}} d \phi \frac{2 m^{2} U+P}{\sqrt{2 m^{2} U+P}} .
$$

Now, the vacua are approached at a finite distance leading to a finite geometric volume

$$
V(P)=\frac{1}{\sqrt{2}} \int_{\phi_{-}}^{\phi_{+}} d \phi \frac{1}{\sqrt{2 m^{2} U+P}} .
$$

As we know this volume is the thermodynamic volume, because

$$
P=-\frac{d E}{d V} .
$$

At the equilibrium $(P=0)$

$$
\phi^{\prime}= \pm m \sqrt{2 U} \Rightarrow \frac{d \phi}{m \sqrt{2 U}}=d x,
$$

where the last formula makes sense only where the field is outside of the vacuum. Then the geometric volume

$$
V=\frac{1}{\sqrt{2}} \int_{\phi_{-}}^{\phi_{+}} \frac{d \phi}{\sqrt{2 m^{2} U}}
$$

can be infinite (usually infinitely extended solitons) or finite (compactons), and is completely governed by the potential or, more precisely, by its behavior close to the vacua.
The volume of a scalar soliton in $(1+1)$-dimensional models is base-space independent. In fact, as the expression of the volume is a target space integral, which obviously is not sensitive to a particular form of the solution, the result is exactly the same, whether the base space is $\mathbb{R}$ or $\mathbb{S}^{1}$ with radius $R$. Of course, the only limitation is that the solution does exist, i.e., the Bogomolny equation has a pertinent soliton on a finite volume space. This is equivalent to the appearance of the Bradlow-type bound. A BPS soliton exists if its geometrical volume is smaller than the volume of the base-space manifold. Quite interestingly, the upper geometric volume is identical to the Bradlow volume (minimal volume in which BPS kinks exist)

$$
V_{B}=\frac{1}{\sqrt{2}} \int_{\phi_{-}}^{\phi_{+}} \frac{d \phi}{\sqrt{2 m^{2} U}} .
$$

Indeed, for compactons one can always put them in a bigger volume $V^{\prime}>V_{B}$ as a superposition of nonoverlapping smaller charge units. This is a rather simple thermodynamical system of a gas of noninteracting compactons. Hence, the bound is now

$$
V_{B} \leq V^{\prime} \leq V_{\mathcal{M}}
$$

If we are below the bound no BPS solitons exist. Topological solitons become now non-BPS objects solving the nonzero-pressure equation.

Let us underline that the Bradlow volume is finite if and only if the kink is a compacton (for example, in $\mathbb{R}$ ). This is uniquely determined by the potential or, more precisely, by the approach to the vacuum. If close to vacuum $\phi=\phi_{a}+$ $\delta \phi$ and $U \propto(\delta \phi)^{c}$, then compactons exist if $c<2$. On the other hand, the Bradlow volume is infinite if and only if kinks are usually infinitely extended solitons in $\mathbb{R}$. Note that in the Abelian-Higgs model at the critical coupling the geometric volume of a BPS vortex on $\mathbb{R}^{2}$ is infinity although the Bradlow volume is finite.

In the next sections we prove that the features outlined above are genuine features of any perfect fluid scalar field theory in any dimension.

\section{B. Higher-dimensional generalization}

There is a generalization of the $(1+1)$-dimensional single scalar theory to any dimension which preserves the perfect fluid property. It relies on the observation that the Lagrangian (4.2) can be written as

$$
\mathcal{L}=-\frac{\left(\phi_{+}-\phi_{-}\right)^{2}}{2} j_{\mu} j^{\mu}-m^{2} U(\phi),
$$

where $j_{\mu}$ is the topological current. Then, in $(d+1)$ dimensions the models we consider are [25] 


$$
\mathcal{L}=-\frac{1}{2} j_{\mu} j^{\mu}-m^{2} U
$$

where now we assume a target space spanned by d scalars $\phi=\left(\phi^{a}\right), a=1 \ldots d$ and

$$
j_{\mu}=\frac{1}{d !} \epsilon^{a_{1} \ldots a_{d}} \epsilon_{\mu \mu_{1} \ldots \mu_{d}} K\left(\phi^{a}\right) \partial^{\mu_{1}} \phi^{a_{1}} \ldots \partial^{\mu_{d}} \phi^{a_{d}},
$$

where $K$ is related to a volume form on the target space $\mathcal{N}$,

$$
d \Omega^{d}=K\left(\phi^{a}\right) d \phi^{1} \wedge \ldots \wedge d \phi^{d} .
$$

For the static case, the total energy is again bounded from below by a Bogomolny-type bound

$$
\begin{aligned}
E & =\frac{1}{2} \int d^{d} x\left(j_{0}^{2}+2 m^{2} U\right) \geq\left|\int d^{d} x j_{0} \sqrt{2 m U}\right| \\
& =k \int_{\mathcal{N}^{\prime}} d \Omega^{d} \sqrt{2 m U},
\end{aligned}
$$

where $k$ is a winding number of the map and $\mathcal{N}^{\prime}$ is the fundamental domain of the soliton, which can but does not have to coincide with the whole target space. In fact, in the examples discussed below we assume $\mathcal{N}=\mathbb{C}$ while $\mathcal{N}^{\prime}=\mathbb{D}$ is a disc. The bound is saturated by solutions of a Bogomolny equation

$$
j_{0}^{2}-2 m^{2} U=0 .
$$

Furthermore, the models lead to a perfect fluid energymomentum tensor that, in the static case, has the nonzero components

$$
\begin{aligned}
& T_{00}=\mathcal{E}=\frac{1}{2} j_{0}^{2}+m^{2} U, \\
& T_{i i}=\mathcal{P}=\frac{1}{2} j_{0}^{2}-m^{2} U,
\end{aligned}
$$

where again due to the energy-momentum tensor conservation law $\mathcal{P}=P=$ const. This gives rise to nonzero pressure configurations that solve the equation

$$
\frac{1}{2} j_{0}^{2}=P+m^{2} U
$$

where the vacuum value must be approached at a finite distance (volume)

$$
V=k \int_{\mathcal{N}^{\prime}} d \Omega^{d} \frac{1}{\sqrt{2 m U+P}}
$$

Finally we can also define the Bradlow volume

$$
V_{B}=k \int_{\mathcal{N}^{\prime}} d \Omega^{d} \frac{1}{\sqrt{2 m U}} .
$$

In the subsequent parts of the paper we provide some examples of such models and discuss them from the Bradlow volume and Bradlow bound perspective.

\section{Global vortices with the SDiff symmetry}

\section{Model and Bogomolny equation}

Perhaps the best and simplest example of a solitonic model with a perfect fluid energy-momentum tensor in $(2+1)$-dimensional space-time is provided by the following model [26],

$$
\mathcal{L}_{\text {BPS vortex }}=\mathcal{L}_{4}+\mathcal{L}_{0},
$$

which is closely related to the so-called BPS baby Skyrme model [27-30]. It consists of two parts, a fourth derivative term

$$
\mathcal{L}_{4}=-\left(u_{\mu} \bar{u}^{\mu}\right)^{2}+u_{\mu}^{2} \bar{u}_{\nu}^{2},
$$

and a nonderivative part, i.e., a potential

$$
\mathcal{L}_{0}=-U(u, \bar{u}),
$$

which has a vacuum at $|u|=1$. Without loss of generality we set all constants equal to 1 . It can be proven that there is a topological bound for the static energy for any potential $U$,

$$
\begin{aligned}
E_{\mathrm{BPS} \text { vortex }}= & \int_{\mathcal{M}} \sqrt{g} d^{2} x\left(\left[(\nabla u \nabla \bar{u})^{2}-(\nabla \bar{u})^{2}(\nabla u)^{2}\right]+U\right) \\
= & \int_{\mathcal{M}} \sqrt{g} d^{2} x\left(\frac{i}{\sqrt{g}} \epsilon_{i j} \partial_{i} u \partial_{j} \bar{u} \pm \sqrt{U}\right)^{2} \\
& \mp 2 i \int_{\mathcal{M}} d^{2} x \epsilon_{i j} \partial_{i} u \partial_{j} \bar{u} \sqrt{U} \\
\geq & \left|2 i \int_{\mathcal{M}} d^{2} x \epsilon_{i j} \partial_{i} u \partial_{j} \bar{u} \sqrt{U}\right| \\
= & 4 \pi|N|\langle\sqrt{U}\rangle_{\mathbb{D}}
\end{aligned}
$$

as the topological charge is

$$
N=\frac{i}{2 \pi} \int d^{2} x \epsilon_{i j} \partial_{i} u \partial_{j} \bar{u} \equiv \int d^{2} x q,
$$

where $q$ is the charge density. The disc $\mathbb{D}=$ $\{u \in \mathbb{C}|| u \mid \leq 1\}$, a subspace of the full $\mathbb{C}$ target space, is the fundamental domain of the vortex solution. Moreover, 


$$
\langle\sqrt{U}\rangle_{\mathbb{D}}=\int \frac{i d u \wedge d \bar{u}}{2 \pi} \sqrt{U}=\frac{1}{\pi} \int f d f d \phi \sqrt{U}
$$

is the average value of $\sqrt{U}$ on $\mathbb{D}$. Here, $f, \phi$ are polar coordinates on $\mathbb{D}$, i.e., $u=f \mathrm{e}^{i \phi}$. The bound is saturated if and only if the following Bogomolny equation is satisfied:

$$
\frac{i}{\sqrt{g}} \epsilon_{i j} \partial_{i} u \partial_{j} \bar{u}= \pm \sqrt{U}
$$

It can be shown that the saturating solutions do fulfil the second-order Euler-Lagrange equations. So, the model possesses a BPS sector, which is not empty if the Bogomolny equation has at least one solution. Note that identically as in the one-dimensional case the Bogomolny equation is defined for any potential. This is a fundamental difference compared to the Abelian-Higgs model. In the subsequent analysis we consider a family of potentials

$$
U=\frac{1}{4}(1-u \bar{u})^{2 \alpha},
$$

where the parameter $\alpha \geq 1 / 2$.

\section{Example-flat space}

Before we discuss the Bradlow bound and the Bradlow volume in a general setup it is instructive to solve the Bogomolny equation exactly in the case where $U$ depends only on $|u|$. In flat space one may perform it assuming the usual axially symmetric ansatz

$$
u(r, \varphi)=f(r) \mathrm{e}^{i N \varphi},
$$

where $N \in \mathbb{Z}$ is the topological charge (winding number) and $f$ is a profile function. Here we used polar coordinates. Note that further (but not necessarily all) solutions can be obtained if SDiff transformations are applied to the axially symmetric solutions. Now, we have

$$
\frac{2 N}{r} f f_{r}=\frac{1}{2}\left(1-f^{2}\right)^{\alpha}
$$

A topologically nontrivial solution requires $f(r=0)=0$ and $f(r=R)=1$ where, as we see, the soliton boundary $R$ can be infinite (as for usual solitons) or finite (compactons). Then, depending on the value of the parameter $\alpha$ we find the following types of solutions. For $\alpha \in\left(\frac{1}{2}, 1\right)$ we have compactons

$1-f^{2}=\left\{\begin{array}{cc}\left(1-\frac{r^{2}}{R^{2}}\right)^{\frac{1}{1-\alpha}} & r \leq R \\ 0 & r \geq R,\end{array} \quad R^{2}=\frac{4 N}{1-\alpha}\right.$.

Here $R$ is the compacton radius. For $\alpha=1$ we find (more than) exponentially localized solitons

$$
1-f^{2}=\mathrm{e}^{-\frac{r^{2}}{4 N}} .
$$

Finally for $\alpha>1$ we obtain powerlike localized solitons

$$
1-f^{2}=\left(\frac{R^{2}}{r^{2}+R^{2}}\right)^{\frac{1}{\alpha-1}}, \quad R^{2}=\frac{4 N}{\alpha-1} .
$$

Here $R$ is just an integration constant (not a compacton radius as the solitons extend to infinity). The conclusion is that in the flat space the BPS sector is not empty. The Bogomolny equation possesses solutions for any value of $\alpha$, i.e., for any possible power-type approach to the vacuum.

\section{Example-finite volume space $\mathcal{M}=\mathbb{S}^{2} \backslash\{(0,0,-1)\}$}

As we found compact vortex solutions for some potentials (some values of $\alpha$ ) on $\mathbb{R}^{2}$, one might naively expect similar compact solutions on manifolds with a finite but sufficiently big volume $\left(V_{\mathcal{M}}>V_{B}\right)$. There is, however, a topological obstruction to the existence of these solutions that requires the manifold to be noncompact, or have a boundary. The reason is that, for vortices, the vacuum is at $|u|=1$, which implies that, outside the domain of the compacton, $u=e^{i \phi\left(x^{j}\right)}$. Further, for vortices with vortex number $N$, the phase $\phi\left(x^{j}\right)$ must change by $2 \pi N$ along closed paths that enclose the domain of the compact vortex. But on a compact manifold without boundary this is impossible. For a manifold with the topology of a two sphere, e.g., one may shrink such a curve to a point in the hemisphere opposite to the compacton, which is obviously incompatible with the nontrivial winding. The way out is a Higgs field $u$ that has a singularity at some point in the "vacuum hemisphere" that impedes the shrinking. This point must then be removed from the manifold, giving it the topology of an open disc. Alternatively, one can remove an open disc from $S^{2}$, leaving a compact manifold with boundary, diffeomorphic to a closed disc. For gauged SDiff vortices, the problem with the nontrivial phase $u=$ $e^{i \phi\left(x^{j}\right)}$ in the vacuum region continues to exist, because it turns out that the magnetic flux of the corresponding BPS solutions is not quantized. We remark that this problem does not exist for BPS baby skyrmions (SDiff baby skyrmions), because there the unique vacuum value is $u=0$, which may be extended to the whole vacuum region (region outside the compacton domain) on arbitrary manifolds. So our considerations below, with some small modifications, apply to that case even for compact manifolds without boundary.

So, let us consider, for simplicity, the two-dimensional sphere with the south pole removed, $\mathcal{M}=\mathbb{S}^{2} \backslash\{(0,0,-1)\}$. The metric still is

$$
d s_{\mathbb{S}^{2}}^{2}=R_{\mathbb{S}^{2}}^{2}\left(d \theta^{2}+\sin ^{2} \theta d \phi^{2}\right),
$$

but now $0 \leq \theta<\pi$. Using the ansatz $u(\theta, \phi)=f(\theta) \mathrm{e}^{i N \varphi}$ we find the Bogomolny equation in the form 


$$
\frac{2 N}{R_{\mathbb{S}^{2}}^{2} \sin \theta} f f_{\theta}=\frac{1}{2}\left(1-f^{2}\right)^{\alpha} .
$$

The boundary conditions are $f(\theta=0)=0$ and $f\left(\theta=\theta_{c}\right)=1$, where now $\theta_{c}$ must be smaller than $\pi$. This is a nontrivial restriction that puts some bounds on the existence of BPS vortices for our equation.

We start with $\alpha \in\left(\frac{1}{2}, 1\right)$. Then,

$$
\begin{aligned}
1-f^{2} & =\left\{\begin{array}{cc}
\left(\frac{\cos \theta-\cos \theta_{c}}{1-\cos \theta_{c}}\right)^{\frac{1}{1-\alpha}} & \theta \leq \theta_{c} \\
0 & \theta \geq \theta_{c},
\end{array}\right. \\
\cos \theta_{c} & =1-\frac{2 N}{R_{\mathbb{S}^{2}}^{2}(1-\alpha)} .
\end{aligned}
$$

This formal solution makes sense only if $1 \geq \cos \theta_{c}>-1$. The first inequality is always satisfied. However, the second one provides a restriction (for a given potential $\alpha<1$ ) for the topological charge and radius of the two sphere, i.e., the area (volume) of the compact manifold

$$
1-\frac{2 N}{R_{\mathbb{S}^{2}}^{2}(1-\alpha)}>-1 \Rightarrow V_{\mathcal{M}}>\frac{4 \pi}{1-\alpha}|N| .
$$

Only for a sphere with the volume larger than $\frac{4 \pi}{1-\alpha}|N|$ a BPS solution with topological charge $N$ exists. This is exactly the Bradlow bound for the SDiff BPS global vortices.

For $\alpha=1$ one can easily solve the BPS equation

$$
1-f^{2}=C \mathrm{e}^{-\frac{R^{2}}{2 N} \cos \theta}
$$

The problem is that there is no $C$ for which the boundary conditions, $f(\theta=0)=0$ and $f\left(\theta=\theta_{c}<\pi\right)=1$, would be satisfied. Therefore the corresponding topologically nontrivial BPS vortex cannot exist. The same happens for any $\alpha>1$. We conclude that for $\alpha \geq 1$ (which for the flat space corresponds to infinitely extended vortices) the BPS sector on $\mathbb{S}^{2} \backslash\{(0,0,-1)\}$ is empty.

\section{The Bradlow bound}

It is not difficult to derive the pertinent Bradlow bound (and corresponding Bradlow volume) for general potential $U$ and any manifold $\mathcal{M}$ with the right topology. Let us assume for the moment that BPS solutions on a given manifold exist. Then we divide the Bogomolny equation (4.37) by $\sqrt{U}$, which makes sense only outside the vacuum

$$
2 \pi \frac{1}{\sqrt{U}} \frac{i}{2 \pi} \frac{1}{\sqrt{g}} \epsilon_{i j} \partial_{i} u \partial_{j} \bar{u}=1
$$

Now we integrate it over the base-space manifold (remembering that the equation is valid only inside the geometric volume of the soliton)

$$
2 \pi \int_{\mathcal{M}} \frac{1}{\sqrt{U}} \frac{i}{2 \pi} \epsilon_{i j} \partial_{i} u \partial_{j} \bar{u} d^{2} x=\int_{\mathcal{M}} \sqrt{g} d^{2} x .
$$

Hence,

$$
2 N \int \frac{1}{\sqrt{U}} \operatorname{vol}_{\mathbb{D}}=V
$$

or

$$
2 \pi N\left\langle\frac{1}{\sqrt{U}}\right\rangle_{\mathbb{D}}=V
$$

where $V$ denotes the geometric volume of the BPS soliton. In fact, the left-hand side of this expression is the Bradlow volume

$$
V_{B}=2 \pi N\left\langle\frac{1}{\sqrt{U}}\right\rangle_{\mathbb{D}} .
$$

Observe that the target space average of $\mathcal{U}^{-1 / 2}$ diverges if the potential gives infinitely extended vortices in flat space, while it is finite in the case of compactons. Furthermore, for compactons it is trivial to construct a solution with the same topological charge but in a bigger volume $V^{\prime}$. One has to put for example $N$ charge one compact vortices such that there is the vacuum between them. Then, such a configuration is still a BPS solution. Obviously, the volume cannot be larger than the volume of the manifold,

$$
V_{B} \leq V^{\prime} \leq V_{\mathcal{M}},
$$

which is precisely the Bradlow bound with the exactly computed Bradlow volume. The energy of all such nonoverlapping BPS vortices is constant, which can be interpreted as a gas of noninteracting solitons with $P=0$. In contrast to the Abelian-Higgs vortices, such BPS configurations with $V>V_{B}$ are trivial to construct.

The meaning of this law is as for the Abelian-Higgs vortices and for kinks in $1+1$ dimensions. An SDiff BPS vortex may exist if its Bradlow volume is smaller that the volume of the base-space manifold; i.e., the manifold $\mathcal{M}$ is large enough to host the BPS vortex. If the Bradlow volume (minimal volume of charge $N$ soliton) $V_{B}$ is bigger that $V_{\mathcal{M}}$, then one can still have vortex solutions on $\mathcal{M}$. However, such vortices are no longer solutions of the Bogomolny equation (not BPS vortices) but of the full EL equations. For the perfect fluid model, the following generalized first-order equation,

$$
2 \pi \frac{i}{2 \pi} \frac{1}{\sqrt{g}} \epsilon_{i j} \partial_{i} u \partial_{j} \bar{u}=\sqrt{U+P},
$$

is a first integral of the static EL equations, where the pressure $P$ is an integration constant. Therefore, 


$$
2 \pi N\left\langle\frac{1}{\sqrt{U+P}}\right\rangle_{\mathbb{D}}=V .
$$

For example, for potentials for which $V_{B}$ is infinite (usually infinitely extended solitons) the SDiff BPS vortices can only be constructed on an infinite volume manifold. If we force them on a finite manifold then they will not be BPS solitons following the Bogomolny equation but vortex solutions of the full EL system, which here means the same as solutions of the generalized Bogomolny equation with nonzero pressure.

So far, the considerations of this section have assumed that solutions to Eq. (4.55) exist (BPS solutions for $P=0$ and non-BPS ones for $P>0$ ). We now show that such solutions do exist, on sufficiently large domains $\mathcal{M}$, under mild topological restrictions. In order to include the case $P=0$, we assume $U$ is chosen so that $V_{B}<\infty$, implying that BPS solutions (if they exist) are compactons. We also restrict to the case $N=1$. Note that (4.49) is just a special case of (4.55), and that the latter has a natural geometric interpretation [29]. Given a pressure $P \geq 0$ solution of the EL equations $u: \mathcal{M} \rightarrow \mathbb{C}$, let $\mathcal{M}_{\text {vac }}=\{x \in \mathcal{M}:|u(x)|=$ 1) and $\mathcal{M}^{\prime}=\mathcal{M} \backslash \mathcal{M}_{\text {vac }}$. Then (4.55) implies that $u$ defines a volume preserving map $\mathcal{M}^{\prime} \rightarrow \mathbb{D}^{\prime}$, where $\mathbb{D}^{\prime}$ is the unit disc equipped with the deformed volume form $\Omega=\frac{i}{2} \frac{d z \wedge d \bar{z}}{\sqrt{U(z, \bar{z})+P}}$. Conversely, given a volume preserving map $u^{\prime}: \mathcal{M}^{\prime} \rightarrow \mathbb{D}^{\prime}$ from $\mathcal{M}^{\prime} \subseteq \mathcal{M}$ of volume $V$, this defines a pressure $P \geq 0$ vortex solution provided it can be continuously extended to $\mathcal{M}$ so that $|u|=1$ on $\mathcal{M}_{\text {vac }}:=\mathcal{M} \backslash \mathcal{M}^{\prime}$.

So, let $\mathcal{M}$ be an oriented Riemannian two-manifold (possibly with boundary) of volume greater than $V$. Then it certainly contains a submanifold $\mathcal{M}^{\prime}$, diffeomorphic to an open disc, with volume $V$. Furthermore, there exists a volume preserving diffeomorphism $u^{\prime}: \mathcal{M}^{\prime} \rightarrow \mathbb{D}^{\prime}$ [31]. Since $u^{\prime}$ has no critical points, it extends uniquely to a map $\bar{u}^{\prime}$ from the closure $\overline{\mathcal{M}}^{\prime}$ to the closure $\overline{\mathbb{D}}$, which maps the boundary $\mathbb{S}^{1}=\partial \mathcal{M}^{\prime}$ homeomorphically, and with winding one, to the unit circle $\mathbb{S}^{1}=\partial \overline{\mathbb{D}}$. As above, let $\mathcal{M}_{\mathrm{vac}}=\mathcal{M} \backslash \mathcal{M}^{\prime}$. Assume that there exists a continuous map $f: \mathcal{M}_{\mathrm{vac}} \rightarrow \partial \mathcal{M}^{\prime}$ such that for all $x \in \partial \mathcal{M}^{\prime}, f(x)=x$. Then the volume preserving map $u^{\prime}: \mathcal{M}^{\prime} \rightarrow \mathbb{D}^{\prime}$ has a continuous extension to $\mathcal{M}$ given by $u(x)=\left(\overline{u^{\prime}} \circ f\right)(x)$ for all $x \notin \mathcal{M}^{\prime}$, which, by definition, has $|u(x)|=1$ for all $x \notin \mathcal{M}^{\prime}$. This, then, is a charge 1 pressure $P \geq 0$ vortex solution on $\mathcal{M}$.

Under what circumstances does a map $f: \mathcal{M}_{\text {vac }} \rightarrow$ $\mathcal{M}_{\text {vac }}$ with the required properties exist? Recall [32] that a retraction of a topological space $X$ to a subspace $A$ is a continuous map $f: X \rightarrow A \subset X$ with $f(x)=x$ for all $x \in A$, which is homotopic to the identity map id: $X \rightarrow X$. Hence, a retraction from $X=\mathcal{M}_{\text {vac }}$ to the subspace $A=\partial \mathcal{M}^{\prime} \subset \mathcal{M}_{\text {vac }}$ certainly has the required property to define an extension of $u^{\prime}$, and so an extension certainly exists if $\mathcal{M}_{\mathrm{vac}}$ retracts to the circle $\partial \mathcal{M}^{\prime}$. This happens if $\mathcal{M} \cong \mathbb{R}^{2}$, since then $\mathcal{M}_{\text {vac }} \cong \mathbb{R}^{2} \backslash \mathbb{D} \cong \overline{\mathbb{D}} \backslash\{0\}$, which retracts (in fact, strongly deformation retracts) to its boundary. The only other case in which a retraction exists is $\mathcal{M} \cong \overline{\mathbb{D}}$, a closed disk, for which $\mathcal{M}_{\mathrm{vac}}$ is homeomorphic to a closed annulus, which, again, strongly deformation retracts to its inner boundary circle. Note, however, that requiring $f: \mathcal{M}_{\mathrm{vac}} \rightarrow \mathcal{M}_{\mathrm{vac}}$ to be a retraction is really overkill: there is no reason to require $f$ to be homotopic to the identity map. Consider the case where $\mathcal{M}$ is compact with boundary. Then $\mathcal{M}$ is diffeomorphic to $S^{2}$ with at least one open disk removed, and some number of handles attached. Remove from $\mathcal{M}$ the open disk $\mathcal{M}^{\prime}$ to obtain $\mathcal{M}_{\text {vac }}$, which is a topologically a sphere with at least two open disks removed, and some number of handles. From $\mathcal{M}_{\text {vac }}$ we can construct a topological space $X$ homeomorphic to a cylinder (equivalently, a closed annulus) as follows: draw a simple closed curve $\gamma$ in the interior of $\mathcal{M}_{\text {vac }}$ that encloses both $\partial \mathcal{M}^{\prime}$ and one of the boundary circles of $\mathcal{M}$. Now define the equivalence relation $\sim$ on $\mathcal{M}_{\text {vac }}$, which collapses all points outside $\gamma$ to a single point. Then $X=\mathcal{M}_{\mathrm{vac}} / \sim$ is homeomorphic to a closed annulus, and the canonical map $\pi: \mathcal{M}_{\mathrm{vac}} \rightarrow X, \pi(x)=[x]$ is tautologically continuous. As previously argued, a retraction $f_{X}$ from $X$ to its inner boundary circle exists. But then $f=f_{X} \circ \pi$ is a continuous map $\mathcal{M}_{\text {vac }} \rightarrow \partial \mathcal{M}^{\prime}$, which restricts to the identity on $\partial \mathcal{M}^{\prime}$, and hence, defines an extension of $u^{\prime}$. The construction is illustrated in Fig. 1. Note that the extension is not unique. In fact, if $\mathcal{M}$ has more than one boundary component, the extension is not even unique up to homotopy, since the curve $\gamma$ can encircle any of the boundary circles. Note also that we can equally well start with $\mathcal{M}$ diffeomorphic to a sphere with at least

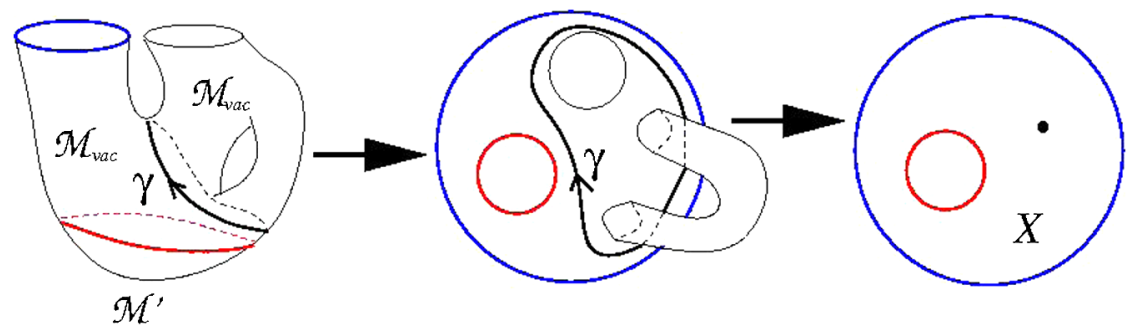

FIG. 1. Extending a volume preserving diffeomorphism $\mathcal{M}^{\prime} \rightarrow \mathbb{D}^{\prime}$ to a global map $\mathcal{M} \rightarrow \overline{\mathbb{D}}$ via a collapse and retraction construction. 
one point (rather than open disc) removed, and any number of handles attached, and take $\gamma$ to enclose exactly one puncture point and $\mathcal{M}^{\prime}$. We then obtain $X$ homeomorphic to a punctured disc which, again, retracts to its boundary. We could also remove some points and some open disks. We see, then, that charge 1 , pressure $P \geq 0$ vortices exist on manifolds $\mathcal{M}$ of sufficiently large volume for essentially any imaginable topology, except compact manifolds without boundary.

From the point of view of the Bradlow bound, there are some similarities between SDiff BPS vortices and AbelianHiggs vortices. There is always a Bradlow volume, i.e., minimal volume for which a BPS solution may exist. If we further decrease the volume of the vortex (by squeezing the solution or by reducing the volume of the manifold) no BPS configurations can exist. Instead, non-BPS vortices may appear, which leads to nonzero pressure. A more careful analysis, however, reveals some interesting differences. First, global SDiff vortices cannot exist on compact manifolds without boundary, because the nontrivial vacuum field $u=e^{i \phi}$ cannot be extended to the whole vacuum region on such manifolds. As said, this problem does not exist for SDiff baby skyrmions and SDiff skyrmions. Secondly, similar to the case of $1+1$ scalar field theories, the geometric volume $V$ of a SDiff BPS vortex is always equal to the Bradlow volume. Therefore, only compactons (whose existence is again completely determined by the potential) lead to a finite Bradlow volume. Thirdly, the (local) existence of a compact vortex on a finite (nonvacuum) domain on any sufficiently large manifold is guaranteed by the fact that the corresponding first-order equation is independent of the metric. All these findings, obviously, continue to hold for further versions of SDiff BPS models like, e.g., the BPS baby Skyrme model or the BPS Skyrme model [33] in 3+1 dimensions. It follows that solutions for these further models exist on arbitrary space manifolds, e.g., on compact manifolds without boundary.

\section{ABELIAN VORTICES WITH THE SDIFF SYMMETRY}

\section{A. Model and Bogomolny equations}

Finally, we extend our analysis for a gauged version of perfect fluid theory that we call the SDiff Abelian vortex model [26]

$$
\begin{aligned}
\mathcal{L}_{\text {gauged BPS vortex }} & \\
= & -\frac{1}{2}\left[\left(D_{\mu} u \overline{D^{\mu} u}\right)^{2}-\left(D_{\mu} u\right)^{2}\left(\overline{D_{\nu} u}\right)^{2}\right]-U(u \bar{u}) \\
& -\frac{1}{4 e^{2}} F_{\mu \nu}^{2},
\end{aligned}
$$

where the usual derivatives are replaced by covariant derivatives given by

$$
D_{\mu} u=u_{\mu}+i A_{\mu} u \text {. }
$$

We now keep the constant $e$ (the dimensionless electric charge). Here we repeat the computation of the topological bound and the corresponding Bogomolny equations saturating the bound [26] (which is a version of the computations originally done for the gauged BPS baby Skyrme model [34]).

The static energy of the SDiff Abelian model is given by

$$
E=\frac{1}{2} \int \sqrt{g} d^{2} x\left(\frac{Q^{2}}{g}+2 U+\frac{1}{e^{2}} \frac{B^{2}}{g}\right),
$$

where the covariant "topological density" $Q$ takes the form

$$
\begin{aligned}
\frac{Q}{\sqrt{g}} & \equiv \frac{i}{\sqrt{g}} \epsilon_{i j} D_{i} u \overline{D_{j} u}=\frac{i}{\sqrt{g}} \epsilon_{i j} u_{i} \bar{u}_{j}-\frac{1}{\sqrt{g}} \epsilon_{i j} A_{i} \partial_{j}|u|^{2} \\
& \equiv 2 \pi \frac{q}{\sqrt{g}}-\frac{1}{\sqrt{g}} \epsilon_{i j} A_{i} \partial_{j}|u|^{2} \\
& =\frac{1}{\sqrt{g}} \epsilon_{i j} \partial_{i}|u|^{2}\left(\phi_{j}+A_{j}\right)=-\frac{1}{\sqrt{g}} \epsilon_{i j} \partial_{i} h\left(A_{j}+\phi_{j}\right),
\end{aligned}
$$

where

$$
u=f \mathrm{e}^{i \phi}, \quad h \equiv 1-f^{2} .
$$

Let us now consider a suitable non-negative expression

$$
\begin{aligned}
0 \leq & \left(\frac{Q}{\sqrt{g}}-w(h)\right)^{2}+\frac{1}{e^{2}}\left(\frac{B}{\sqrt{g}}+b(h)\right)^{2} \\
= & \left(\frac{Q^{2}}{g}+w^{2}\right)+\frac{1}{e^{2}}\left(\frac{B^{2}}{g}+b^{2}\right)-2 \cdot 2 \pi \frac{q}{\sqrt{g}} w \\
& +\frac{2}{\sqrt{g}} \epsilon_{i j} w(h) \partial_{i} h A_{j}+\frac{2}{e^{2}} b(h) \frac{1}{\sqrt{g}} \epsilon_{i j} \partial_{i} A_{j},
\end{aligned}
$$

where $w(h)$ and $b(h)$ are new functions of the target space variable $h$ that are still to be defined. The last two terms in the upper expression can be combined into a total derivative if we assume that these functions obey the following relation:

$$
\begin{aligned}
b(h) & =e^{2} W(h), \quad W(h) \equiv \int_{0}^{h} d h^{\prime} w\left(h^{\prime}\right) \\
& \Rightarrow \epsilon_{i j} w(h) \partial_{i} h A_{j}+\frac{1}{e^{2}} b(h) \epsilon_{i j} \partial_{i} A_{j}=\epsilon_{i j} \partial_{i}\left(W A_{j}\right) .
\end{aligned}
$$

Of course, by construction $W(h)$ is 0 at the vacuum value $h=0$ and therefore the total derivative does not contribute to the energy and may be omitted. The remainder of the non-negative expression may indeed be written as the energy density minus the topological term $2 q W_{h}$ provided that the function $W$ obeys the first-order nonlinear ODE (the "superpotential equation") 


$$
W_{h}^{2}+e^{2} W^{2}=2 U(h) .
$$

Assuming that this is the case we find for the energy the inequality

$$
\begin{aligned}
E= & \frac{1}{2} \int \sqrt{g} d^{2} x\left(\left(\frac{Q}{\sqrt{g}}-W_{h}\right)^{2}+\frac{1}{e^{2}}\left(\frac{B}{\sqrt{g}}-e^{2} W\right)^{2}\right) \\
& +2 \pi\left|\int d^{2} x q W_{h}\right| \geq 2 \pi\left|\int d^{2} x q W_{h}\right| .
\end{aligned}
$$

Hence

$$
E \geq 2 \pi|N|\left\langle W_{h}\right\rangle_{\mathbb{D}}
$$

The bound is saturated if the following Bogomolny equations are satisfied:

$$
\frac{Q}{\sqrt{g}}=W_{h}, \quad \frac{B}{\sqrt{g}}=-e^{2} W .
$$

Again, one can prove that solutions of the Bogomolny equations solve the full second-order field equations.

\section{B. Bradlow bound for the gauged BPS vortices}

For the derivation of the volume of a soliton in the SDiff BPS-type models (nongauge case) we simply integrated the BPS equation over the manifold $\mathcal{M}$. So, one would like to do this also in the gauged version. Unfortunately, as the magnetic and matter (vortex) field are nontrivially mixed in the Bogomolny equations, we were not able to perform this computation in an ansatz-independent manner. However, since the model in the static version has SDiff invariance, a result obtained in a certain ansatz may be easily generalized to any SDiff related configuration, which at the end renders our result ansatz (and coordinate) independent. As in the case of global vortices, we first consider the case of compact solutions on $\mathbb{R}^{2}$, and then invoke the metric independence of the BPS equations (5.13) to argue that the same solutions continue to exist (at least locally, i.e., on a compact nonvacuum domain) on arbitrary (sufficiently large) manifolds for an appropriate choice of coordinates (leading to the same volume element $g$ ).

We consider flat space and assume the ansatz

$$
u(r, \varphi)=f(r) \mathrm{e}^{i N \varphi}, \quad A_{0}=A_{r}=0, \quad A_{\varphi}=N a(r) .
$$

The Bogomolny equations take the following form,

$$
\begin{gathered}
N h_{y}(1+a)=-W_{h}, \\
N a_{y}=-e^{2} W,
\end{gathered}
$$

where, as before, $h=1-f^{2}$ and $y=r^{2} / 2$. First we compute the magnetic flux, which can be expressed in terms of the asymptotic value of the gauge potential,
$\Phi=\int r d r d \varphi B=2 \pi N \int d y a_{y}=2 \pi N a\left(y_{0}\right) \equiv 2 \pi N a_{\infty}$.

Here $y_{0}$ is the position of the soliton boundary, which is finite for compactons and infinite for noncompact solitons. Dividing one Bogomolny equation by the other we get

$$
\frac{a_{y}}{1+a}=e^{2} h_{y} \frac{W}{W_{h}} .
$$

Hence

$$
\partial_{y} \ln (1+a)=e^{2} \partial_{y} F,
$$

where

$$
F_{h} \equiv \frac{W}{W_{h}}
$$

The last expression can be formally integrated,

$$
F(h)=\int_{0}^{h} d h^{\prime} \frac{W\left(h^{\prime}\right)}{W_{h}\left(h^{\prime}\right)},
$$

which results in

$$
\ln C(1+a)=e^{2} F(h(y)),
$$

where $C$ is an integration constant. Here we assume that the first derivative of the superpotential does not possess 0 's in the interval $0<h \leq 1$. This guarantees that $F_{h}$ is finite in the same interval. At the vacuum $h=0$, where $W_{h}=0$, we assume that the potential behaves algebraically, i.e., $V \sim h^{2 \alpha}$ for some $\alpha>0$, then $W_{h} \sim h^{\alpha}, W \sim$ $h^{\alpha+1}$ near $h=0$ and $F_{h}$ is, in fact, 0 at $h=0$. As a consequence, $F$ exists and is finite in the whole interval $h \in[0,1]$. For such (generic) potentials, it follows from the above result that

$$
a(y)>-1, \quad \text { for all } y
$$

and that the limit $a \rightarrow-1$ may be reached only in the limit $e \rightarrow \infty$. The integration constant may be determined from the boundary conditions $h(y=0)=1, a(y=0)=0$,

$$
F(1)=\frac{1}{e^{2}} \ln C \Rightarrow C=\mathrm{e}^{e^{2} F(1)},
$$

which, together with $h\left(y_{0}\right)=0$ and $F(h=0)=0$ leads to the asymptotic expression

$$
a_{\infty}=-1+\mathrm{e}^{-e^{2} F(1)},
$$


which may be inserted into the expression for the magnetic flux

$$
\Phi=2 \pi N\left(-1+\mathrm{e}^{-e^{2} F(1)}\right)
$$

The magnetic flux is not quantized; therefore, these solutions, again, cannot exist on compact manifolds without boundary. They may, however, exist on noncompact manifolds (e.g., the punctured two-sphere) of on manifolds with boundary (e.g., a closed disc).

Note that $F(1)$ (and the flux) can be expressed as the target space averaged value of a function of the superpotential. Indeed,

$$
F(1)=\int_{0}^{1} d h \frac{W(h)}{W_{h}(h)}=\left\langle\frac{W}{W_{h}}\right\rangle_{\mathbb{D}},
$$

which is also valid for any $\mathcal{M}$ that supports solutions, at all.

Now let us continue the computation of the volume of the soliton. The gauge field is

$$
1+a(y)=\mathrm{e}^{e^{2}(F(h(y))-F(h=1))} .
$$

This can be inserted into the first Bogomolny equation

$$
d y=-N \frac{1+a}{W_{h}} d h=-N \frac{\mathrm{e}^{e^{2}(F(h)-F(1))}}{W_{h}} d h
$$

Therefore, after integrating both sides we get

$$
V=2 \pi y_{0}=2 \pi N \mathrm{e}^{-e^{2} F(1)} \int_{0}^{1} \frac{\mathrm{e}^{e^{2} F(h)}}{W_{h}} d h .
$$

As the computation of the volume does not require any particular form of solution, it is completely solution and coordinate independent (which means that also in the gauge case the volume is a thermodynamical function). Owing to the metric independence of the BPS equations it is, therefore, true for any manifold $\mathcal{M}$ with the right size and topology to host solutions. This allows us to write a Bradlow-type relation for the existence of gauged SDiff vortices

$$
V_{B} \leq V^{\prime} \leq V_{\mathcal{M}}
$$

where the Bradlow volume is, once again, equal to the geometric volume of the soliton

$$
V_{B}=2 \pi y_{0}=2 \pi N \mathrm{e}^{-e^{2} F(1)} \int_{0}^{1} \frac{\mathrm{e}^{e^{2} F(h)}}{W_{h}} d h
$$

Let us also comment that the volume of the soliton can be written as an averaged integral

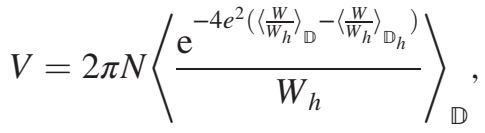

where $\mathbb{D}_{h}$ is a part of the unit disc parametrized by $(\tilde{h}, \phi): \tilde{h} \in[0, h], \phi \in[0,2 \pi]$.

To conclude, both nongauged and gauged SDiff BPS models are very similar. For example, exactly as in the nongauge case, if a potential supports infinitely extended $U(1)$ BPS vortices in flat space then, for any finite-size manifold, the BPS sector is empty.

\section{CONFORMAL SOLITONS}

To complete our investigations we present solitonic models with conformal symmetry. As one could expect, the Bradlow volume takes zero value, which means that the corresponding BPS solitons can be constructed on an arbitrarily small compact manifold.

Let us consider the $C P^{1}$ model

$$
\mathcal{L}=\frac{u_{\mu} \bar{u}^{\mu}}{\left(1+|u|^{2}\right)^{2}}
$$

The BPS sector is defined by the Cauchy-Riemann condition

$$
\bar{\partial} u=0 \quad \text { or } \quad \partial u=0
$$

solved by (anti-) holomorphic functions. Such holomorphic functions can be defined also on a compact manifold, for example, $\mathbb{S}_{R}^{2}$ with an arbitrary radius $R$ [35]. This can be achieved by use of the stereographic projection, which relates coordinates on the two sphere with the usual complex variables $z \in \mathbb{C}$. Since the action (and the Bogomolny equation) is conformally invariant the radius of the base-space sphere can take any value. Therefore, the corresponding Bradlow volume is

$$
V_{B}=0
$$

Note that the analysis is more subtle if the base-space manifold is $T^{2}=\mathbb{S}^{1} \times \mathbb{S}^{1}$. Then the BPS sector is not empty for $N>1$ and is given by the degree $N$ elliptic functions [36]. In the degree one case, it is possible to construct configurations with energy arbitrary close to $2 \pi$ although equality is never attained. A similar situation occurs if we consider the Cauchy-Riemann equation on a disc. There are no nonconstant holomorphic maps satisfying the obvious single point boundary condition. This means that the BPS sector is again empty.

The same concerns the instantons in the self-dual Yang-Mills $S U(2)$ model, which can be constructed on $\mathbb{S}^{4}$ with arbitrary radius. Another example of such conformal solitons (with $V_{B}=0$ ) is provided by pure Skyrme instantons [37]. 


\section{SUMMARY}

In Sec. II, we proved that the geometric volume of a soliton coincides with the thermodynamical volume also for models with local gauge symmetries. This identification holds for base-space manifolds whether they are flat or curved, compact or noncompact.

Then, using this geometric volume, we analyzed the relationship between the soliton volume $V$, the volume of the manifold $V_{\mathcal{M}}$, and the Bradlow volume $V_{B}$, i.e., the minimal volume of the base space for which a given BPS theory may possess a nonempty BPS sector.

We found that the existence of a Bradlow bound seems to be a rather generic feature of BPS solitonic theories. By this we mean the following. Consider a solitonic field theory, which supports BPS solitons in flat (infinite) Minkowski space-time. Then consider the same action but on a manifold $\mathcal{M}$ with finite volume. There is a minimal volume of such a manifold (the Bradlow volume $V_{B}$ ) such that a solution to the (first-order) BPS equations can exist. If the volume of the manifold is further reduced, $V_{\mathcal{M}}<V_{B}$, then solutions cannot be BPS configurations but, rather, solitonic solutions of the full Euler-Lagrange equations. As a consequence, a nonzero pressure emerges as a quantity that characterizes these solutions. All this shows that the Bradlow volume is a rather general concept that may be defined for many BPS models. In Sec. III C 2 we found an exception to this rule, where the formal constant solutions (3.26) have infinite energy, leading to an infinite formal Bradlow volume, whereas the model still supports compact BPS vortices of finite size.

We studied the Bradlow bound both for generalized Abelian-Higgs models and for a certain type of perfect fluid-type models that we called SDiff BPS models (as the static energy functional is invariant under SDiff transformations of the base space), which in $1+1$ dimensions reduce to the usual scalar field theory. In particular, for the SDiff BPS models our findings can be summarized as follows.

(1) The Bradlow volume is equal to the geometric volume of the corresponding BPS soliton $V=V_{B}$. This says also that the geometric volume is base-space independent. Here it is, of course, assumed that the base-space manifold has the required minimal size and the right topology to host BPS solitons.

(2) A finite value of the Bradlow volume is observed if and only if the corresponding BPS solutions in flat Minkowski space-time are compactons. This, on the other hand, is completely controlled by the potential term or, more precisely, by its approach to the vacuum. The usual infinitely extended solitons (again in flat Minkowski space-time) give rise to an infinite value of the Bradlow volume. Note that such a simple relation between the type of solution (compacton/infinitely extended soliton) and the value of the Bradlow volume (finite/ infinite) is not a generic feature. Recall for example the Abelian-Higgs model where solitons in $\mathbb{R}^{2}$ are infinitely extended although the Bradlow volume takes a finite value, leading to the relation $V \geq V_{B}$.

(3) BPS as well as non-BPS solitons are equivalent, whether one considers the model on a compact manifold with boundary or on a finite volume (noncompact) manifold. Therefore, there is no difference between SDiff vortices on $\mathbb{S}^{2} \backslash\{(0,0,-1)\}$ and $\mathbb{D} \subset \mathbb{R}^{2}$. As a consequence, reducing the volume of a manifold $\mathcal{M}$ is equivalent to squeezing the soliton into a smaller volume on the fixed background manifold. This allows for easy access to the mean-field equation of state. Note again that this is different if compared to Abelian-Higgs vortices.

Further, one can use the Bradlow volume to characterize general solitonic models. In fact, we found three types of theories with qualitatively different relations between the geometric volume of a soliton in the BPS sector and the Bradlow volume.

(1) Abelian-Higgs-type models-the BPS soliton always covers the full manifold $\mathcal{M}$, i.e., $V=V_{\mathcal{M}}$. Furthermore, the Bradlow bound requires $V \geq V_{B}$. The inequality is in fact saturated-BPS vortices exist for a compact manifold with $V_{\mathcal{M}}=V_{B}$

(2) Perfect fluid (SDiff) models $-V=V_{B}$, i.e., the geometric (identical to thermodynamical) volume of the BPS soliton is base-space (manifold $\mathcal{M}$ ) independent and obviously $V \leq V_{\mathcal{M}}$. Some generalized Abelian-Higgs models supporting compactons behave in this way, as well.

(3) Conformal models-again the BPS solitons cover the full manifold $\mathcal{M}$, i.e., $V=V_{\mathcal{M}}$. But now $V_{B}=0$.

It is interesting to note that the physical behavior of the different phases is quite different between the AbelianHiggs model and the SDiff models. In the SDiff models, the phase transition only exists for models supporting compactons, and the transition is from a fluid phase with constant nonzero pressure density but nonconstant energy density for $V \leq V_{B}$ to a gas of noninteracting compact vortices for $V>V_{B}$. For the Abelian-Higgs model (and for those generalizations that lead to noncompact BPS vortices), on the other hand, the phase transition is from the constant solution (3.26) with constant energy density for $V \leq V_{B}$ to a BPS solution that always covers the whole base space for $V \equiv V_{\mathcal{M}}>V_{B}$. The case of generalized Abelian-Higgs models supporting compact BPS vortices is somewhere in between, supporting the constant solutions for $V \leq V_{B}$, but supporting compact vortices (with a total volume equal to $V_{B}$ ), surrounded by empty space (vacuum), which fills the remaining volume $V_{\mathcal{M}}-V_{B}$. 
One very important step forward in this thermodynamical/ fluid dynamical analysis of soliton models, obviously, is its generalization to nonzero temperature. This generalization is complicated by the fact that in a classical field theory there are infinitely many degrees of freedom that must be heated up (defrozen). In [38] an approximate but exact equation of state at nonzero-temperature $T$ was derived for the Abelian-Higgs model on a large sphere (corresponding to small $T$ ), by restricting the defreezing to the lightest degrees of freedom (the moduli). A full thermodynamical description at nonzero temperature might well require the quantized version of the soliton model as a starting point. This endeavor, however, is very difficult owing to the perturbative nonrenormalizability of said field theories. Only nonperturbative methods, therefore, have a chance to lead to interesting results.

\section{ACKNOWLEDGMENTS}

C. A. acknowledges financial support from the Ministry of Education, Culture, and Sports, Spain (Grant No. FPA 2014-58-293-C2-1-P), the Xunta de Galicia (Grant No. INCITE09.296.035PR and Conselleria de Educacion), the Spanish Consolider-Ingenio 2010 program Centro Nacional de Fisica de Particulas, Astroparticulas y Nuclear (CPAN) (Grant No. CSD2007-00042), and Fondo Europeo de Desarrollo Regional (FEDER).
[1] N. Manton and P. Sutcliffe, Topological Solitons (Cambridge University Press, Cambridge, 2004).

[2] T. H. R. Skyrme, Proc. R. Soc. A 260, 127 (1961); Nucl. Phys. 31, 556 (1962); J. Math. Phys. 12, 1735 (1971).

[3] V. L. Ginzburg and L. D. Landau, Zh. Eksp. Teor. Fiz. 20, 1064 (1950).

[4] C. Adam, M. Haberichter, and A. Wereszczynski, Phys. Lett. B 754, 18 (2016).

[5] C. Adam, C. Naya, J. Sanchez-Guillen, M. Speight, and A. Wereszczynski, Phys. Rev. D 90, 045003 (2014).

[6] C. Adam, M. Haberichter, and A. Wereszczynski, Phys. Rev. C 92, 055807 (2015).

[7] C. Adam, T. Klähn, C. Naya, J. Sanchez-Guillen, R. Vazquez, and A. Wereszczynski, Phys. Rev. D 91, 125037 (2015).

[8] E. B. Bogomolnyi, Sov. J. Nucl. Phys. 24, 449 (1976).

[9] M. K. Prasad and C. M. Sommerfield, Phys. Rev. Lett. 35, 760 (1975).

[10] S. B. Bradlow, Commun. Math. Phys. 135, 1 (1990).

[11] P. Higgs, Phys. Lett. 12, 132 (1964).

[12] H. B. Nielsen and P. Olesen, Nucl. Phys. B61, 45 (1973).

[13] A. A. Abrikosov, Sov. Phys. JETP 5, 1174 (1957).

[14] J. M. Speight, J. Geom. Phys. 92, 30 (2015).

[15] N. Manton and P. Sutcliffe, Topological Solitons (Cambridge University Press, Cambridge, 2004).

[16] S. M. Nasir, Nonlinearity 11, 445 (1998).

[17] N. Manton, J. Phys. A 50, 125403 (2017).

[18] J. Ambjorn and P. Olesen, Phys. Lett. B 214, 565 (1988); Nucl. Phys. B315, 606 (1989).

[19] S. B. Gudnason and M. Nitta, J. High Energy Phys. 05 (2017) 039.
[20] D. Bazeia, E. da Hora, C. dos Santos, and R. Menezes, Eur. Phys. J. C 71, 1833 (2011).

[21] M. A. Lohe, Phys. Rev. D 23, 2335 (1981).

[22] F. Contatto, Phys. Lett. B 768, 23 (2017).

[23] M. Dunajski, Phys. Lett. B 710, 236 (2012).

[24] D. Bazeia, L. Losano, M. A. Marques, R. Menezes, and I. Zafalan, Eur. Phys. J. C 77, 63 (2017).

[25] C. Adam, L. A. Ferreira, E. da Hora, A. Wereszczynski, and W. J. Zakrzewski, J. High Energy Phys. 08 (2013) 062.

[26] C. Adam, J. Sanchez-Guillen, A. Wereszczynski, and W. J. Zakrzewski, Phys. Rev. D 87, 027703 (2013).

[27] C. Adam, T. Romanczukiewicz, J. Sanchez-Guillen, and A. Wereszczynski, Phys. Rev. D 81, 085007 (2010).

[28] T. Gisiger and M. B. Paranjape, Phys. Rev. D 55, 7731 (1997).

[29] J. M. Speight, J. Phys. A 43, 405201 (2010).

[30] A. N. Leznov, B. Piette, and W. J. Zakrzewski, J. Math. Phys. 38, 3007 (1997).

[31] J. Moser, Trans. Am. Math. Soc. 120, 286 (1965).

[32] A. Hatcher, Algebraic Topology (Cambridge University Press, Cambridge, 2002).

[33] C. Adam, J. Sanchez-Guillen, and A. Wereszczynski, Phys. Lett. B 691, 105 (2010).

[34] C. Adam, C. Naya, J. Sanchez-Guillen, and A. Wereszczynski, Phys. Rev. D 86, 045010 (2012).

[35] J. A. McGlade and J. M. Speight, Nonlinearity 19, 441 (2006); J. M. Speight, J. Geom. Phys. 47, 343 (2003).

[36] J. M. Speight, Commun. Math. Phys. 194, 513 (1998).

[37] J. M. Speight, Phys. Lett. B 659, 429 (2008).

[38] N. Manton, Nucl. Phys. B400, 624 (1993). 